\title{
Towards a Unified Quadrature Framework for Large-Scale Kernel Machines
}

\author{
Fanghui Liu* Xiaolin Huang ${ }^{\dagger} \quad$ Yudong Chen ${ }^{\ddagger} \quad$ Johan A.K. Suykens*
}

November 17, 2020

\begin{abstract}
In this paper, we develop a quadrature framework for large-scale kernel machines via a numerical multiple integration representation. Leveraging the fact that the integration domain and measure of typical kernels, e.g., Gaussian kernels, arc-cosine kernels, are fully symmetric, we introduce a deterministic fully symmetric interpolatory rule to efficiently compute its quadrature nodes and associated weights to approximate such typical kernels. This interpolatory rule is able to reduce the number of needed nodes while retaining a high approximation accuracy. Further, we randomize the above deterministic rule such that the proposed stochastic version can generate dimension-adaptive feature mappings for kernel approximation. Our stochastic rule has the nice statistical properties of unbiasedness and variance reduction with fast convergence rate. In addition, we elucidate the relationship between our deterministic/stochastic interpolatory rules and current quadrature rules for kernel approximation, including the sparse grids quadrature and stochastic spherical-radial rule, thereby unifying these methods under our framework. Experimental results on several benchmark datasets show that our fully symmetric interpolatory rule compares favorably with other representative random features based methods
\end{abstract}

\section{Introduction}

Kernel methods [SS03, SVGDB+02, KE19] have demonstrated to be powerful in statistical machine learning. Key to the kernel methods is the kernel trick for efficiently computing the inner product in a high-dimensional (or even infinite-dimensional) feature space. In particular, the inner product in a reproducing kernel Hilbert space (RKHS) $\mathcal{H}$ can be computed as $k(\boldsymbol{x}, \boldsymbol{y})=\langle\varphi(\boldsymbol{x}), \varphi(\boldsymbol{y})\rangle_{\mathcal{H}}$ using a kernel function $k(\cdot, \cdot): \mathbb{R}^{d} \times \mathbb{R}^{d} \rightarrow \mathbb{R}$. The implicit non-linear mapping $\varphi: \mathcal{X} \rightarrow \mathcal{H}$, which need not be known explicitly, transforms elements of the input space $\mathcal{X}$ into $\mathcal{H}$.

One well-known bottleneck of applying kernel methods is their huge space and time complexities $\left[\mathrm{LHG}^{+} 20\right.$, OG19, $\mathrm{DLG}^{+}{ }^{20]}$. For example, given $n$ observations in $\mathbb{R}^{d}$, kernel ridge regression (KRR) takes about $\mathcal{O}\left(n^{3}\right)$ time for training and $\mathcal{O}\left(n^{2}\right)$ space to store the kernel matrix. To make kernel methods feasible for large scale problems, random Fourier features (RFF) [RR07] provides a practical alternative using an explicit nonlinear mapping $\Phi: \mathbb{R}^{d} \rightarrow \mathbb{R}^{D}$ to approximate a kernel function $k(\cdot, \cdot)$ such that $k(\boldsymbol{x}, \boldsymbol{y}) \approx \Phi(\boldsymbol{x})^{\top} \Phi(\boldsymbol{y})$ for any two samples $\boldsymbol{x}, \boldsymbol{y} \in \mathcal{X}$. Then the linear learning is conducted in the resulting feature space with $\mathcal{O}\left(n D^{2}\right)$ time and $\mathcal{O}(n D)$ memory while preserving the expressive power of nonlinear kernel methods. Therefore, RFF can be used for scaling up traditional kernel methods [LPSS ${ }^{+}$14, SGT18], recent convolutional neural tangent kernel (CNTK) $\left[\mathrm{ADH}^{+} 19 \mathrm{~b}\right]$, and also demonstrates its feasibility to analysis over-parameterized neural networks [Dan17, $\left.\mathrm{ADH}^{+} 19 \mathrm{a}, \mathrm{dRBK} 20\right]$. It spawns the new direction on kernel approximation with a flurry of research papers for variance reduction, including quasi-Monte Carlo (QMC) [YSAM14], orthogonal random features (ORF) [YSC $\left.{ }^{+} 16\right]$, random orthogonal matrices (ROM) [CRW17], and quadrature based methods with sparse grids quadrature (SGQ) [DDSR17] or the stochastic spherical-radial (SSR) rule [MKBO18]. Partly due to

\footnotetext{
*Department of Electrical Engineering (ESAT-STADIUS), KU Leuven. Email: fanghui.liu@kuleuven.be

$\dagger$ Institute of Image Processing and Pattern Recognition, and also with Institute of Medical Robotics, Shanghai Jiao Tong University, Shanghai 200240, P.R. China

${ }^{\ddagger}$ School of Operations Research and Information Engineering, Cornell University, Ithaca, NY 14850 USA
} 


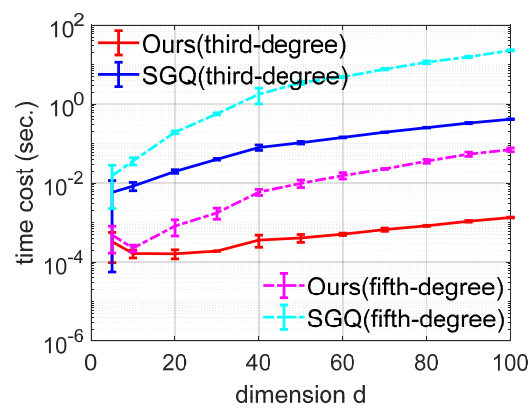

(a) Time cost

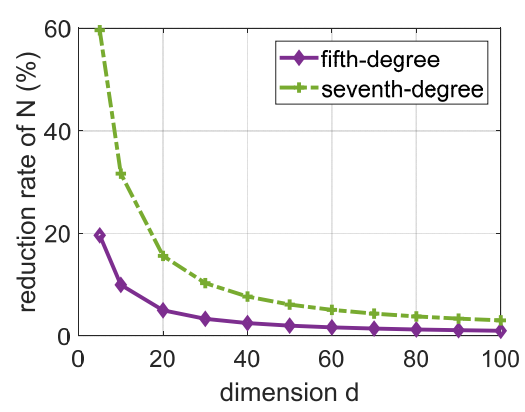

(b) $\frac{N_{\mathrm{SGQ}}-N_{\text {Ours }}}{N_{\text {Ours }}} \times 100 \%$

Figure 1. The benefits of fully symmetric property in our method against SGQ in time cost (a), and the reduction rate of required nodes $(\mathrm{b})$.

its remarkable repercussions, Rahimi and Recht [RR07] won the test-of-time award for their seminal work on RFF at NeurIPS 2017.

In this paper, we focus on kernel approximation in which kernels $k(\cdot, \cdot)$ admit the following $d$-dimensional integral representation $I_{d}$ given by [MKBO18]

$$
k(\boldsymbol{x}, \boldsymbol{y}):=I_{d}\left(f_{\boldsymbol{x} \boldsymbol{y}}\right)=\int_{\mathbb{R}^{d}} p(\boldsymbol{\omega}) f_{\boldsymbol{x} \boldsymbol{y}}(\boldsymbol{\omega}) \mathrm{d} \boldsymbol{\omega},
$$

where the random vector $\boldsymbol{\omega} \in \mathbb{R}^{d}$ is subject to the multivariate normal distribution $\mathcal{N}\left(\mathbf{0}, \boldsymbol{I}_{d}\right)$ and thus $p(\cdot)$ can be regarded as a weighting function. The integrand $f_{\boldsymbol{x} \boldsymbol{y}}$, short for $f$, is defined as $f_{\boldsymbol{x} \boldsymbol{y}}(\boldsymbol{\omega}):=$ $\left\langle\phi\left(\boldsymbol{\omega}^{\top} \boldsymbol{x}\right), \phi\left(\boldsymbol{\omega}^{\top} \boldsymbol{y}\right)\right\rangle$ with a mapping $\phi(\cdot)$. As demonstrated by [Lyu17, MKBO18], various kernel admits this $d$-dimensional integration representation by choosing different $\phi(\cdot)$. For example, the popular Gaussian kernel corresponds to $\phi(x)=[\cos (x), \sin (x)]^{\top}$; the zero-order arc-cosine kernel admits this representation by choosing $\phi(x)$ as the Heaviside function; and the first-order arc-cosine kernel corresponds to $\phi(x)=$ $\max \{0, x\}$.

To approximate the kernel function in Eq. (1.1), such $d$-dimensional integration can be approximated by quadrature rules

$$
k(\boldsymbol{x}, \boldsymbol{y}):=I_{d}\left(f_{\boldsymbol{x} \boldsymbol{y}}\right) \approx \sum_{i=1}^{N} a_{i} f_{\boldsymbol{x} \boldsymbol{y}}\left(\gamma_{i}\right),
$$

where $\gamma_{i} \in \mathbb{R}^{d}$ are called the quadrature nodes and $a_{i} \in \mathbb{R}$ are the corresponding weights. Accordingly, the kernel function can be approximated by a weighted sum of $N$ nodes. Here the nodes and weights can be chosen by various quadrature rules such that there is no approximation error in Eq. (1.2) whenever the integrand $f$ belongs to all polynomials with a total degree up to $2 L-1$, where $L$ is the accuracy level. For example, in the univariate integration, i.e., $d=1$ in Eq. (1.2), Gaussian quadrature (GQ) uses $L$ nodes to deliver the exact value of polynomials up to $(2 L-1)$-degree without approximation error for $\omega_{1}^{i_{1}} \omega_{2}^{i_{2}} \cdots \omega_{d}^{i_{d}}$ with $\sum_{j=1}^{d} i_{j} \leq 2 L-1$. If the integrand $f$ is not a $(2 L-1)$-degree polynomial but has $c$-order bounded derivatives, Gaussian quadrature has approximation error but converges to the true value at a certain $\mathcal{O}\left(N^{-c}\right)$ rate, which is better than $\mathcal{O}(1 / \sqrt{N})$ in the standard Monte Carlo sampling. In multidimensional cases $(d>1)$, the $d$-dimensional integration in Eq. (1.2), conducted by multivariate quadrature, works in the similar way as the univariate case. For instance, Gaussian quadrature directly extends the univariate case to multidimensional cases by product rules but suffers from "curse of dimensionality": the number of required nodes is $N=L^{d}$ in an exponential order of $d$. However, as an advanced quadrature rule, sparse grid quadrature (SGQ) [HW08] uses a linear combination of low-level tensor products of univariate quadrature rules, of which the number of nodes $N$ can be decreased in a polynomial order of $d$. Given an accuracy level $L$, SGQ is also exact for polynomials of the total order up to $2 L-1$.

Recall Eq. (1.1), the integral is not generic but has a nice property: both the integration domain $\mathbb{R}^{d}$ and the Gaussian weighting function $p(\boldsymbol{\omega})$ are fully symmetric. Benefiting from this property, the nodes and the weights can be efficiently obtained from a pre-given vector through permutations and sign changes of 


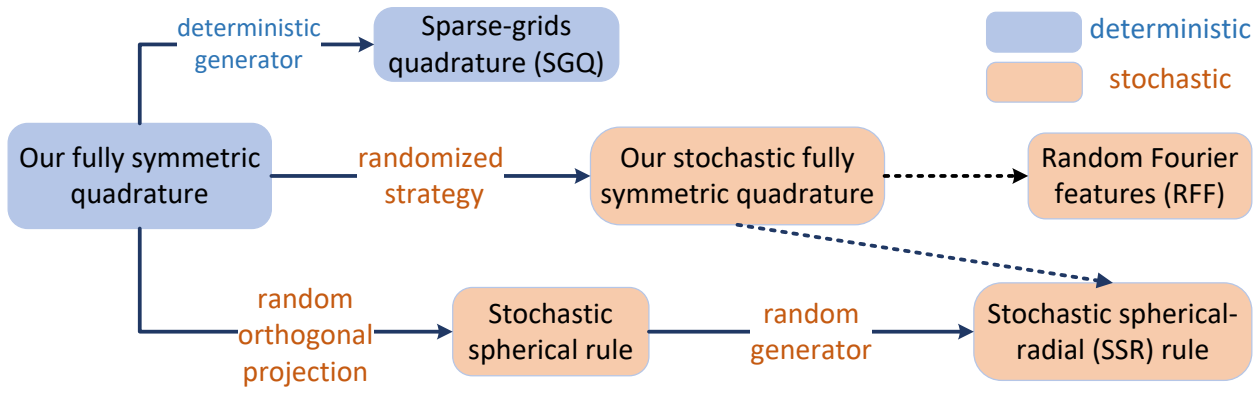

Figure 2: Relationship between quadrature based methods.

its coordinates. Furthermore, such fully symmetric property is helpful to reduce the number of the required nodes $N$ in quadrature rules. For example, considering $d=25$ with an accuracy level $L=4$ for seventhdegree polynomial exactness approximation, Gaussian quadrature requires $4^{25}$ nodes; SGQ needs 24,751 nodes; while the fully symmetric rule needs 22,151 nodes, which reduces over $10 \%$ nodes. And even in some cases, the required nodes can be reduced over 50\% [NR99]. Figure 1 demonstrates the superiority of the fully symmetric rule against SGQ on time cost and the reduction rate of required nodes ${ }^{1}$. One can find that, higher-degree rules results in higher reduction rates on the number of required $N$.

Based on the above analysis, inspired by the deterministic fully symmetric interpolatory rule [GK96], we develop a deterministic scheme to efficiently approximate such $d$-dimensional integration for kernel approximation. Besides, we propose a new stochastic version with unbiased estimation and low variance properties for dimension-adaptive feature mappings. Furthermore, we elucidate the relationship between including SGQ [DDSR17], SSR [MKBO18] and the developed deterministic/stochastic fully symmetric interpolatory rule, thereby unifying these methods under our framework in Figure 2. We make the following contributions:

- we develop a deterministic quadrature rule based on the fully symmetric interpolatory rule for kernel approximation. We derive the third/fifth-degree rules and obtain an extremely sparse feature mapping in $\mathcal{O}(d)$ time and space complexity, see in Section 3.

- we randomize the above deterministic rule and thus propose a new stochastic version for dimensionadaptive feature mappings. We prove that the proposed stochastic fully symmetric interpolatory rule guarantees unbiased estimation for kernel approximation and admits a variance reduction property at a $\mathcal{O}\left(1 / d^{2}\right)$ convergence rate, see in Section 4 .

- we build a unifying quadrature framework for kernel approximation as shown in Figure 2, that unifies our deterministic/stochastic fully symmetric interpolatory rules, SGQ and SSR. We show that i) with a suitable choice of parameters, our deterministic interpolatory rule recovers SGQ; ii) SSR can be regarded as a doubly stochastic version of our deterministic rule: one stochasticity comes from random projection scheme and another source is using a randomized generator; see in Section 5.

Besides, experimental results on several benchmark datasets show that the proposed deterministic/stochastic fully symmetric interpolatory rule achieves a promising quality of kernel approximation and also performs well on classification tasks.

\section{Related Works and Preliminaries}

In this section, we give an overview of representative random features based algorithms for kernel approximation, refer to a survey [LHCS20] for details. Then we briefly introduce the basic ideas behind deterministic fully symmetric interpolatory rules in numerical integration.

\footnotetext{
${ }^{1}$ In third-degree rule, the number of nodes in SGQ and our fully symmetric rule are the same $N=2 d+1$ but are different in higher-degree rules.
} 


\section{$2.1 \quad$ Related Works}

To approximate the kernel function, current kernel approximation methods for finding $\left\{a_{i}, \boldsymbol{\gamma}_{i}\right\}_{i=1}^{N}$ can be divided into Monte Carlo and quadrature based approaches.

Monte Carlo based methods are often equal-weight rules where the nodes $\left\{\gamma_{i}\right\}_{i=1}^{N}$ are obtained by variants of Monte Carlo sampling. For example, the standard random Fourier features (RFF) adopts $\boldsymbol{\gamma}_{i} \equiv \boldsymbol{\omega}_{i} \sim p(\cdot)$ by Monte Carlo sampling and the equal weights $a_{1}=\cdots=a_{N} \equiv 1 / N$. To reduce the approximation variance, orthogonal random features $(\mathrm{ORF})\left[\mathrm{YSC}^{+} 16\right]$ incorporates an orthogonality constraint on the transformation matrix $\boldsymbol{W}=\left[\boldsymbol{\omega}_{1}, \cdots, \boldsymbol{\omega}_{N}\right]$, demonstrated by theoretical guarantees on variance reduction [CRW17]. Sampling theory [Nie92] suggests that the convergence rate of Monte-Carlo used in RFF and ORF can be significantly improved by sampling in a deterministic scheme instead of i.i.d. version. Accordingly, QuasiMonte Carlo (QMC) sampling [ASYM16], as a possible middle-ground method, utilizes a low-discrepancy sequence for sampling, which achieves faster convergence rates $\mathcal{O}\left(\log ^{d} N / N\right)$. In fact, a series of empirical and theoretical results [Lyu17, CRCW19] have demonstrated that, coupling samples to be orthogonal to one another (i.e., uniformly distributed over the space), rather than being i.i.d., can significantly improve statistical efficiency. The above used sampling methods are data-independent. Another line in random features based algorithms is to utilize data-dependent sampling strategy for better approximation quality and generalization properties. Typical examples include leverage score based sampling [AKM $\left.{ }^{+} 17\right]$, fast leverage score approximation [RCCR18, $\left.\mathrm{LHC}^{+} 20\right]$, Christoffel functions [PBV18], and Fourier sparse leverage scores [EMM20].

In quadrature based methods, the nodes are usually given by deterministic rules (can be extended to a stochastic version) and the weights are often not equal. Examples include Gaussian quadrature [Eva93] and sparse grids quadrature (SGQ) [DDSR17] based on the Smolyak formula [HW08]. Instead of directly approximating the $d$-dimensional integration, the stochastic spherical-radial (SSR) rule [GM98] transforms the integration in Eq. (1.1) to a double-integral over the unit $d$-sphere and over the radius, which are then approximated by a stochastic spherical rule and a stochastic radial rule, respectively. This rule has been successfully applied to kernel approximation [MKBO18] and achieves promising approximation quality.

If the integrand $f(\boldsymbol{\omega})$ belongs to a RKHS, the above polynomial quadrature schemes can be termed as kernel-based quadrature, such as kernel herding [BLJO12], Bayesian quadrature [BOC ${ }^{+} 17, \mathrm{KS} 18$, GS18], and leverage-score quadrature [Bac17]. Note that, kernel quadrature is in essence different from the previously studied polynomial quadrature in functional spaces, model formulation, and scope of application.

\subsection{Preliminaries: Deterministic Fully Symmetric Interpolatory Rules}

Here we briefly introduce the class of fully symmetric interpolatory rules [GK96]. It is based on fully symmetric sets [MS67, Coo97], where point sets are obtained from a given vector through permutations and sign changes of its coordinates.

To introduce the fully symmetric interpolatory rule, we need the following definitions. Denote an integervalued vector $\boldsymbol{p}=\left[p_{1}, p_{2}, \cdots, p_{d}\right]$ with $p_{i} \in\{0,1, \ldots, m\}$, and define $\mathcal{P}^{(m, d)}$ as a set of all distinct $d$-partitions of the integers $\{0,1, \ldots, m\}$, i.e.

$$
\mathcal{P}^{(m, d)}=\left\{\boldsymbol{p} \in \mathbb{N}^{d} \mid p_{1} \geq p_{2} \geq \cdots \geq p_{d} \geq 0,\|\boldsymbol{p}\|_{1} \leq m\right\} .
$$

For example, when $m=2, d=4$, we have

$$
\mathcal{P}^{(2,4)}=\{(0,0,0,0),(1,0,0,0),(2,0,0,0),(1,1,0,0)\} .
$$

It shows that any finite fully symmetric set can be decomposed into a finite number of disjoint subsets admitting that any member of a particular subset can be used to generate the whole subset. Then, let the node $\boldsymbol{\lambda}_{\boldsymbol{p}}=\left[\lambda_{p_{1}}, \lambda_{p_{2}}, \cdots, \lambda_{p_{d}}\right]^{\top}$ be real, distinct, and nonnegative, with $\lambda_{0}=0$. In quadrature rules, $\boldsymbol{\lambda}_{\boldsymbol{p}}$ is also termed as the generator, and accordingly the fully symmetric basic rule $f\left(\boldsymbol{\lambda}_{\boldsymbol{p}}\right)$ is given by

$$
f\left(\boldsymbol{\lambda}_{\boldsymbol{p}}\right)=\sum_{q \in \Pi_{p}} \sum_{\boldsymbol{\nu} \in \mathcal{V}_{d}} f\left(\nu_{1} \lambda_{q_{1}}, \nu_{2} \lambda_{q_{2}}, \ldots, \nu_{d} \lambda_{q_{d}}\right),
$$


where in the outer sum, $\Pi_{p}$ denotes the set of all permutations of $\boldsymbol{p}$. In the inner sum, $\mathcal{V}_{d}$ is the set of all vectors with the form $\boldsymbol{\nu}=\left[\nu_{1}, \nu_{2}, \cdots, \nu_{d}\right]$ with $\nu_{i}= \pm 1$ for those $i$ with $\lambda_{i} \neq 0$. For example, when $d=4$ and $\boldsymbol{p}=(2,0,0,0)$

$$
\begin{aligned}
f\left(\boldsymbol{\lambda}_{\boldsymbol{p}}\right)= & f\left( \pm \lambda_{2}, 0,0,0\right)+f\left(0, \pm \lambda_{2}, 0,0\right) \\
& +f\left(0,0, \pm \lambda_{2}, 0\right)+f\left(0,0,0, \pm \lambda_{2}\right) .
\end{aligned}
$$

Based on the above definitions, the $d$-dimensional integration in Eq. (1.1) can be approximated by a rule of the total polynomial degree up to $2 m+1$

$$
I_{d}(f) \approx Q^{(m, d)}(f)=\sum_{\boldsymbol{p} \in \mathcal{P}^{(m, d)}} a_{\boldsymbol{p}}^{(m, d)} f\left(\boldsymbol{\lambda}_{\boldsymbol{p}}\right)
$$

where the weight $a_{\boldsymbol{p}}^{(m, d)}$ is

$$
a_{\boldsymbol{p}}^{(m, d)}=2^{-K} \sum_{\|\boldsymbol{u}\|_{1} \leq m-\|\boldsymbol{p}\|_{1}} \prod_{i=1}^{d} \frac{b_{u_{i}+p_{i}}}{\prod_{j=0, \neq p_{i}}^{u_{i}+p_{i}}\left(\lambda_{p_{i}}^{2}-\lambda_{j}^{2}\right)},
$$

where $\boldsymbol{u}=\left[u_{1}, u_{2}, \cdots, u_{d}\right]$ is the set of the integers $\{0,1, \ldots, m\}$. If $\boldsymbol{q}$ is one of the permutations of $\boldsymbol{p}$, then $a_{\boldsymbol{q}}^{(m, d)}=a_{\boldsymbol{p}}^{(m, d)}$. And $K$ is the number of nonzero components in $\boldsymbol{p}$ and $b_{i}$ satisfies

$$
b_{i}=\frac{1}{\sqrt{2 \pi}} \int_{-\infty}^{+\infty} e^{-x^{2} / 2} \prod_{j=0}^{i-1}\left(x^{2}-\lambda_{j}^{2}\right) \mathrm{d} x \quad(i \geq 1),
$$

with $b_{0}=1$. We can compute $b_{1}=1$ and $b_{2}=3-\lambda_{1}^{2}$.

Accordingly, $Q^{(m, d)}(f)$ stands for the weighted sum of evaluations of $f$ at the nodes of the fully symmetric set on the distinct $d$-partitions $\mathcal{P}^{(m, d)}$. The theory for fully symmetric interpolatory rules [GK96] shows that $Q^{(m, d)}(f)$ is an approximation to $I_{d}(f)$ that is exact for all polynomials with the total degree $2 m+1$ or less. That means, $m+1$ is the accuracy level that defined in GQ and SGQ before. Note that, although the mathematical foundations and derivations of the fully symmetric rule are relatively complex, the obtained feature mapping for kernel approximation in this paper is quite simple and easy to be implemented when the nodes and weights are given (or even directly obtained by a look-up table). We will illustrate this in the next section.

Different from previous works: When compared to the original work on fully symmetric interpolatory rules, [GK96, GM98], the contribution of this paper lies in the developed deterministic and stochastic kernel approximation schemes, the statistical properties of our stochastic rule, and casting quadrature rules in a unifying framework.

Besides, both our method and [KS18] shares the basic idea on the deterministic fully symmetric rule [GK96] but are totally different in the following aspects: 1) the function space of the integrand $f$ is different: $f$ in [KS18] is modeled as Gaussian process and belongs to RKHS; while $f$ in our method is actually an activation function mapping from $\mathbb{R}^{d}$ to $\mathbb{R}$. 2) the formulation is different: the fully symmetric structure in our model naturally stems from symmetry of the underlying measure and the integration domain, which is different from [KS18] that is about to construct fully symmetric sets in quite sophisticated schemes. Besides, the weights in [KS18] are chosen to minimize the posterior variance of the integral; while the weights in ours are directly computed by orthogonal polynomials. 3) the scope of application is different: Bayesian quadrature in [KS18] cannot be used for kernel approximation.

\section{Deterministic Rules for Kernel Approximation}

In this section, we present our third-degree and fifth-degree deterministic fully symmetric rules for kernel approximation.

In the deterministic fully symmetric interpolatory rule [GK96], $Q^{(m, d)}(f)$ is a weighted sum of fully symmetric basic rules $f\left(\boldsymbol{\lambda}_{\boldsymbol{p}}\right)$. So the kernel $k$ (a.k.a. the $d$-dimensional integration) in Eq. (1.2) can be 
approximated by a weighted sum of evaluations of $f$ at the nodes of the fully symmetric set on the distinct $d$-partitions $\mathcal{P}^{(m, d)}$

$$
k(\boldsymbol{x}, \boldsymbol{y}) \approx Q^{(m, d)}(f)=\sum_{\boldsymbol{p} \in \mathcal{P}^{(m, d)}} a_{\boldsymbol{p}}^{(m, d)} f\left(\boldsymbol{\lambda}_{\boldsymbol{p}}\right),
$$

where the weights $a_{\boldsymbol{p}}^{(m, d)}$ and generator $\boldsymbol{\lambda}_{\boldsymbol{p}}$ play significant roles in quadrature rules. Different generation schemes for $\boldsymbol{\lambda}_{\boldsymbol{p}}$ lead to various quadrature based approaches. For example, SGQ [DDSR17] uses deterministic values to generate $\boldsymbol{\lambda}_{\boldsymbol{p}}$; while $\boldsymbol{\lambda}_{\boldsymbol{p}}$ in SSR [MKBO18] is sampled from a probability distribution. Our fully symmetric interpolatory rule follows with [GK96] that selects $\boldsymbol{\lambda}_{\boldsymbol{p}}$ in a deterministic scheme. We will detail their relations in Section 5.

Third-degree rule: Based on Eq. (3.1), we present the third-degree rule (i.e., $m=1)$ to approximate the kernel $k$ in Eq. (1.1) such that $k(\boldsymbol{x}, \boldsymbol{y}) \approx Q^{(1, d)}(f)$

$$
Q^{(1, d)}(f)=a_{0}^{(1, d)} f(\mathbf{0})+a_{1}^{(1, d)} \sum_{i=1}^{d}\left[f\left(\lambda_{1} \boldsymbol{e}_{i}\right)+f\left(-\lambda_{1} \boldsymbol{e}_{i}\right)\right]
$$

where $\boldsymbol{e}_{i}$ is a unit vector with the $i$-th element being 1 . The weights are given by $a_{0}^{(1, d)}=1-d / \lambda_{1}^{2}$ and $a_{1}^{(1, d)}=1 /\left(2 \lambda_{1}^{2}\right)$ in Eq. (2.2). Finally, our third-degree rule outputs $\left\{a_{i}, \gamma_{i}\right\}_{i=0}^{2 d}$ with

$$
\left\{\begin{aligned}
\gamma_{i} & =\mathbf{0}_{d \times 1} ; a_{i}=1-d / \lambda_{1}^{2} ; i=0 \\
\gamma_{i} & =\lambda_{1} \boldsymbol{e}_{i} ; a_{i}=1 / 2 \lambda_{1}^{2} ; 1 \leq i \leq d \\
\gamma_{i} & =-\lambda_{1} \boldsymbol{e}_{i} ; a_{i}=1 / 2 \lambda_{1}^{2} ; d+1 \leq i \leq 2 d,
\end{aligned}\right.
$$

which results in the number of nodes $N=2 d+1$ in the third-degree rule. The generator $\boldsymbol{\lambda}=\left[\lambda_{0}, \lambda_{1}\right]^{\top}$ with $\lambda_{0}=0$ usually selects $\lambda_{1}$ by successive extensions of the one-dimensional 3-point Gauss-Hermite rule so that certain sets of weights vanish, i.e., $\lambda_{1}=\sqrt{3}$.

Feature mapping: We finally obtain the explicit feature mapping by the third-degree rule for kernel approximation

$$
\Phi(\boldsymbol{x})=\left[\sqrt{a_{0}} \phi\left(\boldsymbol{\gamma}_{0}^{\top} \boldsymbol{x}\right), \sqrt{a_{1}} \phi\left(\boldsymbol{\gamma}_{1}^{\top} \boldsymbol{x}\right), \cdots, \sqrt{a_{2 d}} \phi\left(\boldsymbol{\gamma}_{2 d}^{\top} \boldsymbol{x}\right)\right]^{\top},
$$

such that $k(\boldsymbol{x}, \boldsymbol{y}) \approx Q^{(m, d)}(f)=\langle\Phi(\boldsymbol{x}), \Phi(\boldsymbol{y})\rangle$. Here the weight $a_{0}=1-d / \lambda_{1}^{2}$ might be negative, but we consider the complex number $\sqrt{a_{0}}$ as usual. It can be observed that, this generation process $\left\{a_{i}, \gamma_{i}\right\}_{i=0}^{2 d}$ is data-independent and deterministic. In kernel approximation, the transformation matrix $\boldsymbol{W}=\left[\gamma_{0}, \gamma_{1}, \cdots, \gamma_{2 d}\right] \in \mathbb{R}^{d \times(2 d+1)}$ can be obtained by Eq. (3.3)

$$
\boldsymbol{W}=\left[\begin{array}{cccccccc}
0 & -\lambda_{1} & \lambda_{1} & 0 & 0 & \cdots & 0 & 0 \\
0 & 0 & 0 & -\lambda_{1} & \lambda_{1} & \cdots & 0 & 0 \\
\vdots & \vdots & \vdots & \vdots & \vdots & \ddots & \vdots & \vdots \\
0 & 0 & 0 & 0 & 0 & \cdots & -\lambda_{1} & \lambda_{1}
\end{array}\right]
$$

For better illustration of our method, here we take the Gaussian kernel $k(\boldsymbol{x}, \boldsymbol{y})=\exp \left(-\|\boldsymbol{x}-\boldsymbol{y}\|_{2}^{2} / 2 \sigma^{2}\right)$ as an example for kernel approximation and discuss the difference with RFF. According to Eq. (1.1), the Gaussian kernel can be approximated by $k(\boldsymbol{x}, \boldsymbol{y}) \approx \sum_{i=1}^{D} a_{i} \cos \left(\boldsymbol{\omega}_{i}^{\top}(\boldsymbol{x}-\boldsymbol{y})\right)$ with the transformation matrix $\boldsymbol{W}=\left[\boldsymbol{\omega}_{1}, \cdots, \boldsymbol{\omega}_{D}\right] \in \mathbb{R}^{d \times D}$ with $D$ features as follows.

In RFF, $a_{i} \equiv 1 / D$ and $W_{i j} \sim \mathcal{N}\left(0,1 / \sigma^{2}\right)$ by Monte Carlo sampling; while in our interpolatory rule, $D$ is equivalent to $N=2 d+1$. The weights $a_{i}$ and the transformation matrix $\boldsymbol{W} \in \mathbb{R}^{d \times(2 d+1)}$ are deterministic, given by Eq. (3.3). Note that, in our model, $\boldsymbol{W}$ is extremely sparse, i.e., has only $2 d$ non-zero elements with $\pm \lambda_{1}$. Accordingly, generating $\boldsymbol{W}$ needs space and time cost $\mathcal{O}(d)$. This is better than RFF, which requires $\mathcal{O}(D d)$ space and time complexity. More specifically, when $d$ is given, the nodes, the weights, and the transformation matrix in our deterministic rule can be directly determined, see Eqs. (3.3) and (3.5). That means, our deterministic rule can be much more efficient for kernel approximation when a look-up table is applied. Besides, as mentioned before, the formulation of the final feature mapping in Eq. (3.4) for kernel approximation is quite simple and easy to be implemented according to Eqs. (3.3) and (3.5). 
Fifth-degree rule: When choosing $m=2$ in Eq. (3.1), we obtain a fifth-degree rule $Q^{(2, d)}$ with $\|\boldsymbol{p}\|_{1} \leq 2$ to further improve the approximation accuracy. To derive the fifth-degree rule, we cast it to three cases, i.e., $\|\boldsymbol{p}\|_{1}=0,\|\boldsymbol{p}\|_{1}=1$, and $\|\boldsymbol{p}\|_{1}=2$. Nevertheless, the derivation of fifth-degree rules is relatively complex, and we put it in Appendix A. In the fifth-degree rule, the number of required nodes in our fully symmetric rule is $N=1+2 d^{2}$, which is less than SGQ with $1+2 d^{2}+2 d$. Further, the feature mapping in our fifth-degree rule can be obtained in a similar way with that of the third-degree rule, and we omit it here.

Convergence rates of approximation error: Since the fully symmetric interpolatory rule can be regarded as a special case of sparse grids quadrature (we will detail this in Section 5), the convergence results of SGQ [Gar12] can be directly applied to it: $\left\|Q^{(m, d)}(f)-I_{d}(f)\right\|=\mathcal{O}\left(N^{-\Theta}\right)$, where the norm is a specific $L_{2}$-norm or $L_{\infty}$-norm and $\Theta$ is the convergence rate, determined by $m, d$, and certain smoothness conditions, refer to [Gar12] for details. More importantly, if we define $N_{\min }(2 m+1, d)$ as the minimal number of nodes needed by $Q^{(m, d)}(f)$ admitting a total degree $2 m+1$ of exactness, our interpolatory rule reduces $N_{\min }(2 m+1, d)$ when compared to the standard SGQ due to the fully symmetric property, see Theorem 1 in [HN07].

\section{Stochastic Rules and its properties}

The above fully symmetric interpolatory rules are determinstic. That means, given $d$, the number of generated nodes $N$ is fixed, which is infeasible to tune $N$ for dimension-adaptive feature mappings in kernel approximation. This is a common issue in deterministic rules, e.g., SGQ [DDSR17]. To tackle this issue in kernel approximation, we revise the randomization method [GM98] for deterministic interpolatory rules and propose a new stochastic version so as to achieve a tight approximation variance. Since current quadrature based methods, e.g., SGQ [DDSR17] and SSR [MKBO18], adopt the third-degree rule instead of higherdegree rules due to sufficient approximation and efficient computation [AH09], we consider the third-degree stochastic rule in this paper. In fact, our results can be extended to the fifth-degree stochastic rule under suitable modifications.

\subsection{Formulation of stochastic rules}

We introduce the random vector $\boldsymbol{\omega} \sim \mathcal{N}\left(\mathbf{0}, \boldsymbol{I}_{d}\right)$ to $Q^{(m, d)}(f)$ in Eq. (3.1). Define a surrogate stochastic rule

$$
M^{(m, d)}(f, \boldsymbol{\omega})=\sum_{\boldsymbol{p} \in P^{(m, d)}} \tilde{a}^{(m, d)}(\boldsymbol{\omega}) f\left(\boldsymbol{\lambda}_{\boldsymbol{p}}\right),
$$

where the nodes $\boldsymbol{\lambda}_{\boldsymbol{p}}$ are the same as that of deterministic rules in Eq. (3.1). However, the weight $\tilde{a}_{\boldsymbol{p}}^{(m, d)}(\boldsymbol{\omega})$ is different from the deterministic one in Eq. (2.2), and is actually a function of the random vector $\boldsymbol{\omega}$

$$
\tilde{a}_{\boldsymbol{p}}^{(m, d)}(\boldsymbol{\omega})=\left\{\begin{array}{l}
1 ; \text { if }\|\boldsymbol{p}\|_{1}=0 \text { and }\|\boldsymbol{u}\|_{1}=0 \\
\frac{2^{-K}}{d} \sum_{\|\boldsymbol{u}\|_{1} \leq m-\|\boldsymbol{p}\|_{1}} \sum_{i=1}^{d} \frac{\prod_{j=0}^{u_{i}+p_{i}-1}\left(\omega_{i}^{2}-\lambda_{j}^{2}\right)}{\prod_{j=0, \neq p_{i}}^{u_{i}+p_{i}}\left(\lambda_{p_{i}}^{2}-\lambda_{j}^{2}\right)} ; \quad \text { otherwise },
\end{array}\right.
$$

where $K$ is the number of nonzero components in $\boldsymbol{p}$. Accordingly, by randomizing the weights, the stochastic version of the third-degree deterministic rule $Q^{(m, d)}(f)$ in Eq. (3.2) is given by

$$
M^{(1, d)}(f, \boldsymbol{\omega})=\tilde{a}_{0}^{(1, d)} f(\mathbf{0})+\tilde{a}_{1}^{(1, d)} \sum_{i=1}^{d}\left[f\left(\lambda_{1} \boldsymbol{e}_{i}\right)+f\left(-\lambda_{1} \boldsymbol{e}_{i}\right)\right],
$$

where the weight is a function of $\boldsymbol{\omega}$, i.e.,

$$
\left\{\begin{array}{l}
\tilde{a}_{0}^{(1, d)}(\boldsymbol{\omega}) \equiv \tilde{a}_{0}^{(1, d)}=1-\sum_{i=1}^{d} \omega_{i}^{2} / \lambda_{1}^{2} \\
\tilde{a}_{1}^{(1, d)}(\boldsymbol{\omega}) \equiv \tilde{a}_{1}^{(1, d)}=\sum_{i=1}^{d} \omega_{i}^{2} / 2 d \lambda_{1}^{2}
\end{array}\right.
$$


Accordingly, the feature mapping associated with $M^{(1, d)}(f)$ is

$$
\widetilde{\Phi}(\boldsymbol{x})=\left[\sqrt{\tilde{a}_{0}(\boldsymbol{\omega})} \phi\left(\boldsymbol{\gamma}_{1}^{\top} \boldsymbol{x}\right), \cdots, \sqrt{\tilde{a}_{2 d}(\boldsymbol{\omega})} \phi\left(\boldsymbol{\gamma}_{2 d}^{\top} \boldsymbol{x}\right)\right]^{\top},
$$

where $\boldsymbol{\gamma}$ is given by Eq. (3.3) and $\boldsymbol{\omega} \sim \mathcal{N}\left(\mathbf{0}, \boldsymbol{I}_{d}\right)$. So $M^{(1, d)}(f, \boldsymbol{\omega})$ is a randomized rule such that $M^{(1, d)}(f, \boldsymbol{\omega}) \approx$ $\langle\widetilde{\Phi}(\boldsymbol{x}), \widetilde{\Phi}(\boldsymbol{y})\rangle$. Unfortunately, $\mathbb{E}_{\boldsymbol{\omega}}\left[M^{(1, d)}(f, \boldsymbol{\omega})\right]=Q^{(1, d)}(f) \neq I_{d}(f)$ is not an unbiased estimator of $I_{d}(f)$.

To pursue an unbiased estimator, by virtue of the classical control variate technique [RM85], we define

$$
r_{m}(f, \boldsymbol{\omega})=f(\boldsymbol{\omega})-M^{(m, d)}(f, \boldsymbol{\omega})+I_{d}\left[M^{(m, d)}(f, \boldsymbol{\omega})\right],
$$

which is still an integration rule for $I_{d}(f)$ of the total polynomial degree $2 m+1$. Hence, our stochastic fully symmetric interpolatory rule is defined as $R_{m}(f)$ such that

$$
k(\boldsymbol{x}, \boldsymbol{y}) \approx R_{m}(f):=\frac{1}{D} \sum_{i=1}^{D} r_{m}\left(f, \boldsymbol{\omega}_{i}\right),
$$

where $\left\{\boldsymbol{\omega}_{i}\right\}_{i=1}^{D} \sim \mathcal{N}\left(\mathbf{0}, \boldsymbol{I}_{d}\right)$. Then by defining $\varphi(\boldsymbol{x})=1 / \sqrt{D}\left[\phi\left(\boldsymbol{\omega}_{1}^{\top} \boldsymbol{x}\right), \cdots, \phi\left(\boldsymbol{\omega}_{D}^{\top} \boldsymbol{x}\right)\right]^{\top} \in \mathbb{R}^{D}$, the final feature mapping associated with $R_{m}(f)$ is dimension-adaptive, which is given by

$$
\widehat{\Phi}(\boldsymbol{x})=\left[\varphi(\boldsymbol{x})^{\top},\left(\frac{\mathrm{j}}{D} \sum_{i=1}^{D} \widetilde{\Phi}\left(\boldsymbol{x}, \boldsymbol{\omega}_{i}\right)\right)^{\top}, \Phi(\boldsymbol{x})^{\top}\right]^{\top} \in \mathbb{R}^{D+4 d+2},
$$

where $\left\{\boldsymbol{\omega}_{i}\right\}_{i=1}^{D} \sim \mathcal{N}\left(\mathbf{0}, \boldsymbol{I}_{d}\right)$, the symbol $\mathrm{j}$ is called an imaginary unit, and the mappings $\widetilde{\Phi}\left(\boldsymbol{x}, \boldsymbol{\omega}_{i}\right)$ and $\Phi(\boldsymbol{x})$ are given by Eqs. (4.2) and (3.4), respectively. As a consequence, we have $k(\boldsymbol{x}, \boldsymbol{y}) \approx\langle\widehat{\Phi}(\boldsymbol{x}), \widehat{\Phi}(\boldsymbol{y})\rangle$.

Remark: We make the following two remarks.

1) In spite of $\widehat{\Phi}(\boldsymbol{x}) \in \mathbb{R}^{D+4 d+2}$, the feature mappings $\widetilde{\Phi}\left(\boldsymbol{x}, \boldsymbol{\omega}_{i}\right)$ and $\Phi(\boldsymbol{x})$ in Eq. (4.5) have only $2 d$ non-zero elements. The nodes are independent of the sampling process and can be pre-given by Eq. (3.3). Accordingly, the space complexity in our stochastic rule is $\mathcal{O}(D d+4 d)$, and thus our stochastic method achieves the same space and time complexity $\mathcal{O}(D d)$ with RFF.

2) Sampling $\left\{\boldsymbol{\omega}_{i}\right\}_{i=1}^{D} \sim \mathcal{N}\left(\mathbf{0}, \boldsymbol{I}_{d}\right)$ is not limited to the standard Monte Carlo sampling. It can be extended to other advanced approaches, e.g., ORF, QMC, SSR, as an alternative way, which is able to pursue further variance reduction. Our experimental results also verify this, see Section 6.3 for details.

\subsection{Statistical Properties}

This subsection elucidates that i) our third-degree stochastic interpolatory rule is unbiased, see in Theorem 1; ii) exhibits a low variance property in Theorem 2 .

Theorem 1. (Unbiased estimation) The stochastic fully symmetric interpolatory rule $R_{m}(f)$ in Eq. (4.4) is an unbiased $(2 m+1)$-degree rule for $I_{d}(f)$ in Eq. (1.1).

Proof Refer to Appendix B.1.

Remark: We have $Q^{(1, d)}(f)=\mathbb{E}_{\boldsymbol{\omega} \sim p(\cdot)}\left[M^{(1, d)}(f, \boldsymbol{\omega})\right]$, and thus $M^{(1, d)}(f, \boldsymbol{\omega})$ is an asymptotically unbiased estimator of $I_{d}(f)$.

Based on the above unbiased estimation, we derive the variance of our third-degree stochastic interpolatory rule for Gaussian kernel approximation. Before proceeding, we introduce some notations and definitions. When discussing the Gaussian kernel $k(\boldsymbol{x}, \boldsymbol{y})=\exp \left(-\|\boldsymbol{x}-\boldsymbol{y}\|_{2}^{2} / 2 \sigma^{2}\right)$, we use the convenient shorthands $\boldsymbol{z}:=(\boldsymbol{x}-\boldsymbol{y}) / \sigma$ and $z:=\|\boldsymbol{z}\|_{2}$. For an algorithm $A$ with $\left\{\boldsymbol{\omega}_{i}\right\}_{i=1}^{D}$ sampled from the distribution $p(\cdot)$, we define its expectation $\mathbb{E}[A]:=\mathbb{E}_{\boldsymbol{\omega} \sim p}\left[1 / D \sum_{i=1}^{D} \cos \left(\boldsymbol{\omega}_{i}^{\top} \boldsymbol{z}\right)\right]$ and variance $\mathbb{V}[A]:=\mathbb{V}_{\boldsymbol{\omega} \sim p}\left[1 / D \sum_{i=1}^{D} \cos \left(\boldsymbol{\omega}^{\top} \boldsymbol{z}\right)\right]$.

Theorem 2. (Lower variance) For the Gaussian kernel $k(\boldsymbol{x}, \boldsymbol{y})=\exp \left(-\|\boldsymbol{x}-\boldsymbol{y}\|_{2}^{2} / 2 \sigma^{2}\right)$, denoting $Q:=$ $Q^{(1, d)}(f)$ for notational simplicity, then the variance of our third-degree stochastic interpolatory rule in Eq. (4.4) is

$$
\mathbb{V}\left[R_{1}(f)\right]-\mathbb{V}[R F F]=\frac{2}{D d}\left(\left[(1-Q)-\frac{1}{2} z^{2} e^{-\frac{z^{2}}{2}}\right]^{2}-\frac{1}{4} z^{4} e^{-z^{2}}\right)
$$


where $\mathbb{V}[R F F]=\left(1-e^{-z^{2}}\right)^{2} / 2 D$ is given by $\left[Y S C^{+} 16\right]$. Specifically, since $Q$ is the approximation of $I_{d}(f)=k(\boldsymbol{x}, \boldsymbol{y}) \in[0,1]$ for the Gaussian kernel, we can also consider $Q^{(1, d)}(f) \in[0,1],{ }^{2}$ then

$$
\mathbb{V}\left[R_{1}(f)\right]-\mathbb{V}[R F F]<0 \text { when } 1-Q<z^{2} e^{-\frac{z^{2}}{2}} .
$$

Proof Refer to Appendix B.2.

Remark: The condition $1-Q<z^{2} e^{-\frac{z^{2}}{2}}$ in Eq. (4.7) is roughly equivalent to $z \leq 1.57$, which actually holds for most cases as demonstrated in Appendix B.2.3. In fact, this condition can be easily satisfied when we normalize $z:=\|\boldsymbol{x}-\boldsymbol{y}\|_{2} / \sigma$ to $z:=\|\boldsymbol{x}-\boldsymbol{y}\|_{2} / \sqrt{d \sigma^{2}}$ by a scaling factor $\sqrt{d}$. This operation is fair since it is quite common in high-dimensional statistics and random matrix theory [EK10, DW18, LR20], and our experiments employ this setting.

Here we compare it with other representative methods on the estimated variance reduction. Variance of $\mathbf{O R F}\left[\mathrm{YSC}^{+} 16\right]$ is bounded by

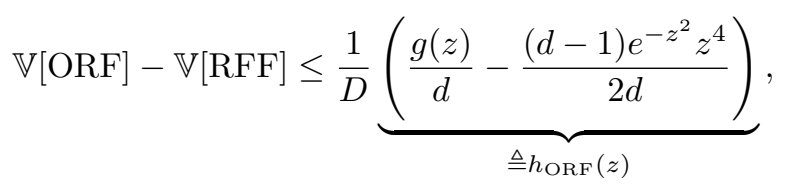

with the function $g(z)=e^{z^{2}}\left(z^{8}+6 z^{6}+7 z^{4}+z^{2}\right) / 4+e^{z^{2}} z^{4}\left(z^{6}+2 z^{4}\right) / 2 d$. It can be found that, the function $g(z)$ actually increases in the exponential order of $z$, which leads to a loose bound for variance estimation. Further, Choromanski et al. [CRCW19] provide a refined analysis on orthogonal Monte Carlo sampling including ORF and ROM, the variance reduction of which is guaranteed at a certain $\mathcal{O}\left(d^{-3 / 2}\right)$ rate, slower than our $\mathcal{O}\left(d^{-2}\right)$ rate.

Variance of SSR [MKBO18] is bounded by

$$
\mathbb{V}[\mathrm{SSR}]-\mathbb{V}[\mathrm{RFF}] \leq \frac{1}{D} \underbrace{\left(\frac{8 d+12}{d-2}-\frac{\left(1-e^{-z^{2}}\right)^{2}}{2}\right)}_{\triangleq h_{\mathrm{SSR}}(z)>0}=\mathcal{O}\left(\frac{1}{d}\right) .
$$

We find that, i) the above inequality for SSR [MKBO18] cannot strictly guarantee $\mathbb{V}[\mathrm{SSR}]<\mathbb{V}[\mathrm{RFF}]$ due to the positive $h_{\mathrm{SSR}}(z)$. While our theoretical result in Theorem 2 admits $\mathbb{V}\left[R_{1}(f)\right]<\mathbb{V}[\mathrm{RFF}]$ under the condition in Eq. (4.7). ii) The variance of SSR converges at the $\mathcal{O}(1 / d)$ rate. Instead, by the sample average of $r_{1}(f, \boldsymbol{\omega})$ in Eq (4.3), our stochastic interpolatory rule $R_{1}(f)$ achieves a tighter approximation variance than SSR and a faster $\mathcal{O}\left(1 / d^{2}\right)$ convergence rate for variance reduction.

\section{Relationship between Quadrature Methods}

In this section, we investigate the relationship between our third-degree deterministic/stochastic fully symmetric interpolatory rules, SGQ [DDSR17], and SSR [MKBO18]. Subsequently, we cast them in our unifying framework for kernel approximation.

\subsection{Relations to the third-degree SGQ rule}

The sparse grids used in [DDSR17] is based on the Smolyak rule [HW08] with accuracy level $L \in \mathbb{C}$ for a $d$-dimensional integration. It can be approximated by a sequence of nested univariate quadrature rules in a tensor product fashion, i.e.

$$
I_{d}(f) \approx A_{d, L}(f)=\sum_{q=0}^{L-1} \sum_{i \in \mathcal{C}_{q}^{d}}\left(\Delta_{i_{1}} \otimes \cdots \otimes \Delta_{i_{d}}\right)(f),
$$

\footnotetext{
${ }^{2}$ Even if the estimation $Q^{(1, d)}(f)$ is out of $[0,1]$, we can still set it to $[0,1]$ by a threshold operator.
} 
with the index vector $\boldsymbol{i}=\left[i_{1}, i_{2}, \cdots, i_{d}\right]$. The set $\mathcal{C}_{q}^{d}=\left\{\boldsymbol{i} \in \mathbb{N}^{d}: \sum_{j=1}^{d} i_{j}=d+q\right\}$ determines the possible accuracy level $i_{j}$ for each univariate quadrature and the nonnegative $q$ prescribes the range of the accuracy level $i_{j}$ in each dimension. $V_{i_{j}}$ is the univariate quadrature rule with the accuracy level $i_{j} \in \boldsymbol{i}$, which generates the difference $\Delta_{i}(f)=V_{i}(f)-V_{i-1}(f), \forall i \in \mathbb{N}$. This rule is a weighted sum of product rules with different combinations of accuracy levels $\boldsymbol{i}$.

To investigate the relationship between the third-degree SGQ and our third-degree fully symmetric interpolatory rule, we construct the third-degree SGQ in Eq. (5.1) using the symmetric univariate quadrature point set $\left\{-\hat{p}_{1}, 0, \hat{p}_{1}\right\}$ with the weight $\left(\hat{a}_{1}, \hat{a}_{0}, \hat{a}_{1}\right)$

$$
I_{d}(f) \approx\left(1-d+d \hat{a}_{0}\right) f(\mathbf{0})+\hat{a}_{1} \sum_{j=1}^{d}\left[f\left(\hat{p}_{1} \boldsymbol{e}_{j}\right)+f\left(-\hat{p}_{1} \boldsymbol{e}_{j}\right)\right] .
$$

If the nodes and their associated weights in the third-degree SGQ are chosen by the following scheme

$$
\hat{a}_{0}:=1-\frac{1}{\lambda_{1}^{2}}, \hat{p}_{1}:=\lambda_{1}, \hat{a}_{1}=\frac{1}{2 \lambda_{1}^{2}},
$$

then SGQ is equivalent to our deterministic fully symmetric interpolatory rule in Eq. (3.2), as shown in Figure 2. In this situation, the fully symmetric interpolatory rule can be regarded as a special case of SGQ [HN07].

\subsection{Relations to the third-degree SSR rule}

In the stochastic spherical-radial rule [GM98], the key step is a change of variable from the Cartesian vector $\boldsymbol{\omega} \in \mathbb{R}^{d}$ to a radius $r$ and direction vector $\boldsymbol{z} \in \mathbb{R}^{d}$. Let $\boldsymbol{\omega}=r \boldsymbol{z}$ with $\boldsymbol{z}^{\top} \boldsymbol{z}=1$, so that $\boldsymbol{\omega}^{\top} \boldsymbol{\omega}=r^{2}$ for $r \in[0, \infty)$, we have

$$
I_{d}(f)=\frac{(2 \pi)^{-\frac{d}{2}}}{2} \int_{U_{d}} \int_{-\infty}^{\infty}|r|^{d-1} e^{-\frac{r^{2}}{2}} f(r \boldsymbol{z}) \mathrm{d} \sigma(\boldsymbol{z}) \mathrm{d} r
$$

where $\sigma(\cdot)$ is the spherical surface measure or the area element on $U_{d}$. SSR includes the following two stochastic integration rules.

stochastic radial rule: $R(h)=\sum_{i=1}^{\hat{N}} \hat{a}_{i} \frac{h\left(\rho_{i}\right)+h\left(-\rho_{i}\right)}{2}$ has the form of the weighted symmetric sums and approximate the infinite range integral $T(h)=\int_{-\infty}^{\infty} e^{-\frac{r^{2}}{2}}|r|^{d-1} h(r) \mathrm{d} r$. In its third-degree rule with $\hat{N}=1$, the node $\rho$ admits $\rho \sim \chi(d+2)$.

stochastic spherical rule: $S_{\boldsymbol{Q}}(s)=\sum_{i=1}^{\bar{N}} \bar{a}_{i} s\left(\boldsymbol{Q} \boldsymbol{z}_{j}\right)$, with a random orthogonal matrix $\boldsymbol{Q}$, approximate an integral of a function $s(\boldsymbol{z})$ over the surface of unit $d$-sphere $U_{d}$. Its third-degree rule can be constructed as

$$
I_{\boldsymbol{Q}, U_{d}}(s)=\frac{\left|U_{d}\right|}{2 d} \sum_{j=1}^{d}\left[s\left(\boldsymbol{Q} \boldsymbol{e}_{j}\right)+s\left(-\boldsymbol{Q} \boldsymbol{e}_{j}\right)\right],
$$

where $\left|U_{d}\right|=2 \Gamma^{d}(1 / 2) / \Gamma(d / 2)=2 \sqrt{\pi^{d}} / \Gamma(d / 2)$ is the surface area of the unit sphere with the Gamma function $\Gamma$. Finally, the third-degree SSR rule in [MKBO18] combines the above two stochastic integrals

$$
I_{d}(f) \approx f(\mathbf{0})\left(1-\frac{d}{\rho^{2}}\right)+\sum_{j=1}^{d} \frac{f\left(-\rho \boldsymbol{Q} \boldsymbol{e}_{j}\right)+f\left(\rho \boldsymbol{Q} \boldsymbol{e}_{j}\right)}{2 \rho^{2}},
$$

with $\rho \sim \chi(d+2)$. And specifically, the random orthogonal matrix $\boldsymbol{Q}$ is substituted by butterfly matrices for space efficiency in their implementation.

Based on the above description, we find that there are two differences between the deterministic fully symmetric interpolatory rule and the SSR rule: one is the way of projection and the other is the selection scheme of the generator. For projection, our deterministic/stochastic interpolatory rules avoid constructing 
Table 1: Relationship between kernel approximation methods.

\begin{tabular}{cc}
\hline Methods & Parameters in Eq. (5.3) \\
\hline SSR & $\beta:=d$ \\
\hline$M^{(1, d)}(f)$ in Eq. (4.1) & $\rho:=\lambda_{1}^{2}$ and $\boldsymbol{Q}:=\boldsymbol{I}$ \\
\hline ORF & $\beta:=d$ and $\rho \sim \chi(d)$ \\
\hline$Q^{(1, d)}(f)$ in Eq. (3.2) & $\rho:=\lambda_{1}^{2}, \boldsymbol{Q}:=\boldsymbol{I}$, and $\beta:=d$ \\
\hline SGQ & $\rho:=\lambda_{1}^{2}, \boldsymbol{Q}:=\boldsymbol{I}, \beta:=d$ \\
& $\left\{\hat{a}_{0}, \hat{p}_{0}, \hat{a}_{1}\right\} \leftarrow \lambda_{1}$ \\
\hline
\end{tabular}

a random orthogonal matrix, which is not feasible to a high dimension $d .^{3}$ For generator, our deterministic/stochastic interpolatory rules use a deterministic one instead of a randomized one. This is because, in a high-degree rule, it requires a large number of random generators and nodes to capture the underlying distribution [KS18].

Here we firstly study the relationship between our deterministic interpolatory rule and the stochastic spherical rule, and then investigate the relationship between our rule and SSR.

Theorem 3. The third-degree stochastic spherical integration rule in Eq. (5.2) can be obtained by the random orthogonal projection of our third-degree fully symmetric interpolatory rule in Eq. (3.2).

Proof Refer to Appendix C.

Accordingly, SSR can be obtained by our deterministic interpolatory rule in Eq. (3.2) with the following two randomized steps. i) random projection: by projecting our third-degree interpolatory rule to the spherical surface of $U_{d}$ with a uniform random orthogonal matrix $\boldsymbol{Q}$, we can obtain the third-degree stochastic spherical rule. ii) random generator: the deterministic generator $\lambda_{1}$ in Eq. (3.2) by Gaussian quadrature is substituted by a random variable $\rho$ with $\rho \sim \chi(d+2)$. By doing so, we can transform our interpolatory rule in Eq. (3.2) to SSR in Eq. (5.2), as shown in Figure 2. Similarly, in Eq. (5.2), if the random orthogonal matrix $\boldsymbol{Q}$ is chosen as the identity matrix, and the parameter $\rho$ equals to $\rho:=\lambda_{1}$, then the SSR rule is transformed to our deterministic fully symmetric interpolatory rule.

Apart from the relations between our deterministic rule and SSR, here we also study the relationship between our stochastic interpolatory rule and SSR. On the one hand, in Eq. (4.3), if we only consider $r_{m}(f, \boldsymbol{\omega})=f(\boldsymbol{\omega})$, and thus our stochastic interpolatory rule degenerates to RFF with the standard Monte Carlo sampling scheme. In the other hand, [MKBO18] point out that RFF or ORF can be regarded as a special case of the stochastic spherical-radial rule. Hence, our stochastic interpolatory rule and SSR can be connected together by RFF. Furthermore, if we only consider $M^{(1, d)}(f, \boldsymbol{\omega})$ in our stochastic interpolatory rule in Eq. (4.3), after the above two stochastic operations (random projection and random generator), it is a triple-stochastic rule with the following formulation

$$
I_{d}(f) \approx f(\mathbf{0})\left(1-\frac{\beta}{\rho^{2}}\right)+\frac{\beta}{d} \sum_{j=1}^{d} \frac{f\left(-\rho \boldsymbol{Q} \boldsymbol{e}_{j}\right)+f\left(\rho \boldsymbol{Q} \boldsymbol{e}_{j}\right)}{2 \rho^{2}},
$$

where $\beta \sim \chi(d), \rho \sim \chi(d+2)$, and $\boldsymbol{Q}$ is a random orthogonal matrix. Specifically, we can easily verify that it is an unbiased estimator of $I_{d}(f)$. The deterministic fully symmetric interpolatory rule, ORF, SGQ, and the SSR rule can be regarded as some special cases of Eq. (5.3) with different parameters, see in Table 1.

\section{Empirical Results}

In this section, we empirically compare our deterministic/stochastic interpolatory rule with several representative approaches for kernel approximation, and then incorporate them into the kernel ridge regression (KRR) for classification on several benchmark datasets. Given nodes and weights in Eq (3.3), our algorithm is straightforward to be implemented for the feature mapping in (3.4) by our deterministic rule and Eq. (4.5) by our stochastic rule. We implement them in MATLAB and carry out on a PC with Intel ${ }^{\circledR}$ i7-8700K CPU $(3.70 \mathrm{GHz})$ and $64 \mathrm{~GB}$ RAM. The source code of our implementation will be public.

\footnotetext{
${ }^{3}$ Even if we consider Hadamard matrices for acceleration, not every $4 d$-dimensional matrix admits a corresponding Hadamard matrix [HS79].
} 

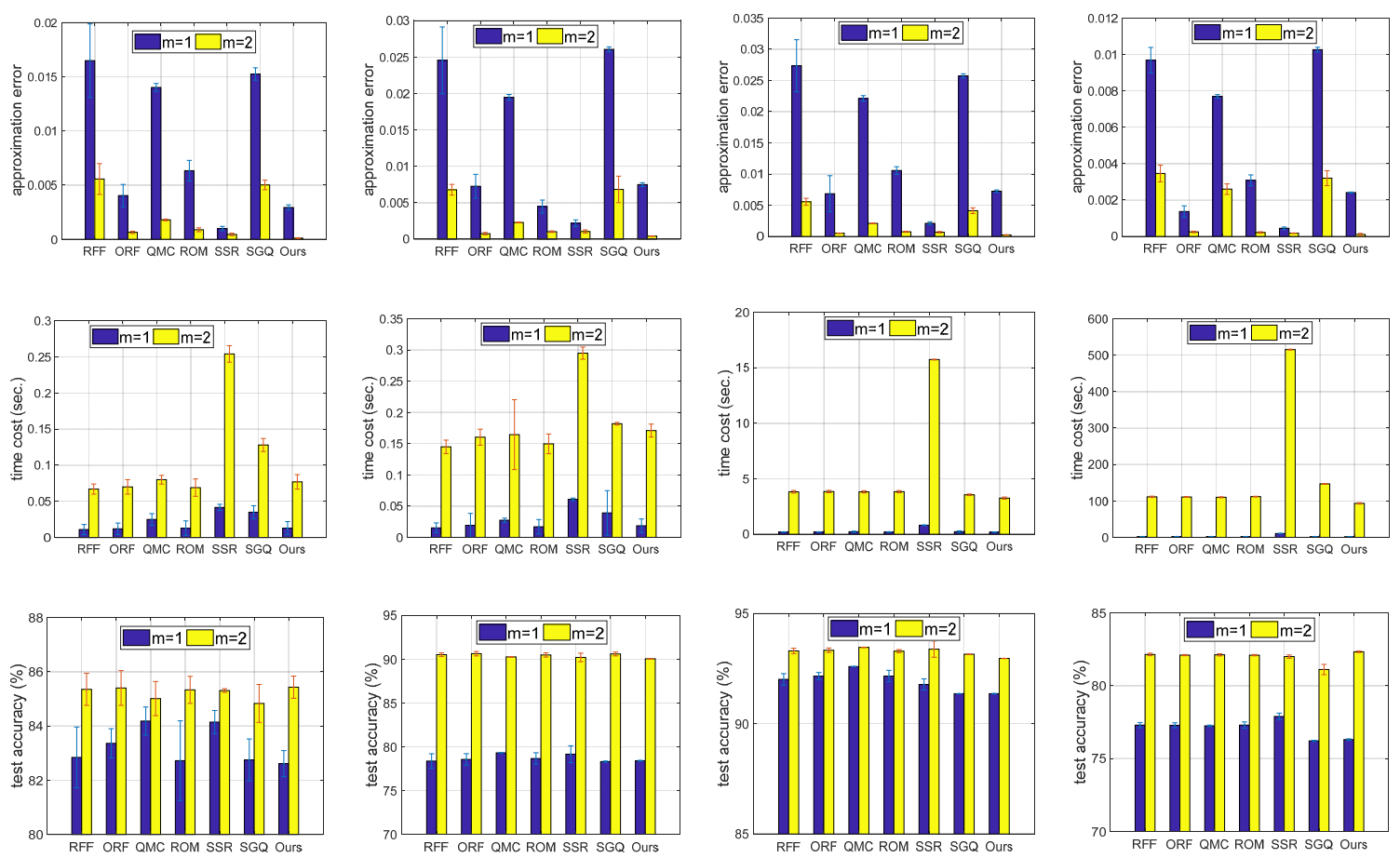

(i) $\mathrm{magicO}_{4}$

(j) letter

(k) ijcnn1

(1) covtype

Figure 3. Results on the RBF kernel in terms of approximation error (top), time cost (middle), and test accuracy (bottom).

Table 2: Dataset statistics and the number of nodes in fifth-degree rules.

\begin{tabular}{cccc|cc}
\hline \multirow{2}{*}{ datasets } & \multirow{2}{*}{$d$} & \multirow{2}{*}{ \#training $\#$ test } & \multicolumn{2}{|c}{ \#nodes $N$} \\
\cline { 5 - 6 } & & & & SGQ & Ours \\
\hline magic04 & 10 & 9,510 & 9,510 & 221 & 201 \\
\hline letter & 16 & 12,000 & 6,000 & 545 & 513 \\
\hline ijcnn1 & 22 & 49,990 & 91,701 & 1013 & 969 \\
\hline covtype & 54 & 290,506 & 290,506 & 5941 & 5833 \\
\hline
\end{tabular}

\subsection{Experimental settings}

Kernel: According to the integral representation in Eq. (1.1), we choose the popular Gaussian kernel and the first-order arc-cosine kernel for experimental validation. Here we use the following formulation of the Gaussian kernel

$$
k(\boldsymbol{x}, \boldsymbol{y})=\exp \left(-\frac{\|\boldsymbol{x}-\boldsymbol{y}\|_{2}^{2}}{2 d \sigma^{2}}\right)
$$

where the feature dimension $d$ is introduced into the kernel width for scaling that follows with [JŞS ${ }^{+} 20$, EK10, MKBO18], as suggested by Theorem 2. The parameter $\sigma^{2}$ is tuned via 5 -fold inner cross validation over a grid of $\{0.1,0.5,1,2,5,10\}$.

The used first-order arc-cosine kernel [CS09] is given by

$$
k(\boldsymbol{x}, \boldsymbol{y})=\frac{1}{\pi}\|\boldsymbol{x}\|_{2}\|\boldsymbol{y}\|_{2}(\sin \theta+(\pi-\theta) \cos \theta),
$$

with $\theta=\cos ^{-1}\left(\frac{\boldsymbol{x}^{\top} \boldsymbol{y}}{\|\boldsymbol{x}\|_{2}\|\boldsymbol{y}\|_{2}}\right)$. In this case, the nonlinear mapping $\phi\left(\boldsymbol{\omega}^{\top} \boldsymbol{x}\right)=\max \left\{0, \boldsymbol{\omega}^{\top} \boldsymbol{x}\right\}$ in Eq. (1.1) is actually a ReLU activation function. 
Datasets: We consider four typical classification datasets including magic04, letter, ijcnn1, and covtype; see Table 2 for an overview. These datasets can be downloaded from https://www.csie.ntu.edu.tw/ cjlin/ libsvmtools/datasets/ or the UCI Machine Learning Repository ${ }^{4}$. We normalize all data sets to $[0,1]^{d}$ and use the pre-given training/test partition on the letter and ijcnn1 data sets. For the remaining two datasets, we randomly pick half of the data for training and the rest for test.

Compared methods: We compare the proposed deterministic/stochastic fully symmetric interpolatory rule with the following algorithms:

- RFF/MC [RR07]: The transformation matrix $\boldsymbol{W}_{\mathrm{RFF}}$ is constructed by the standard Monte Carlo sampling scheme with $W_{i j} \sim \mathcal{N}\left(0,1 / d \sigma^{2}\right)$ in Eq. (6.1) for Gaussian kernel approximation and $W_{i j} \sim$ $\mathcal{N}(0,1)$ for the first-order arc-cosine kernel approximation.

- $\mathrm{ORF}\left[\mathrm{YSC}^{+} 16\right]$ : The transformation matrix $\boldsymbol{W}_{\mathrm{ORF}}$ is constructed by a random orthogonal matrix with $\boldsymbol{W}=\boldsymbol{D} \boldsymbol{Q}$, where $\boldsymbol{D}$ is a diagonal matrix with diagonal elements sampled from the $\chi(d)$ distribution and $\boldsymbol{Q}$ is obtained from the QR decomposition of $\boldsymbol{W}_{\mathrm{RFF}}$. Note that, this approach can be applied to the arc-cosine kernel in practice but lacks theoretical guarantees.

- ROM [CRW17]: The transformation matrix $\boldsymbol{W}_{\mathrm{ROM}}$ is constructed by a series of structural random orthogonal matrices with $\boldsymbol{W}=c \prod_{i=1}^{t} \boldsymbol{H} \boldsymbol{D}_{i}$, where $\boldsymbol{H}$ is a normalized Hadamard matrix and $\boldsymbol{D}_{i}$ is the Rademacher matrix with $\mathbb{P}\left(D_{i i}= \pm 1\right)=1 / 2$. Here $c$ is chosen as $\sqrt{2 / \sigma^{2}}$ for Gaussian kernel approximation and $\sqrt{d}$ for arc-cosine kernel approximation.

- $\mathrm{QMC}$ [ASYM16]: The transformation matrix $\boldsymbol{W}_{\mathrm{QMC}}$ is constructed by a deterministic low-discrepancy Halton sequence.

- SGQ [DDSR17]: This is a deterministic method that generates fixed-size feature mappings, which is constructed by the nodes and the weights. To make SGQ adaptively tune the feature dimension, we follow with [DDSR17] and subsample the grid nodes according to the distribution determined by their weights such that the mapping feature dimension is $D$.

- SSR [MKBO18]: The feature mapping is constructed by the third-degree stochastic spherical-radial rule. The required random orthogonal matrix $\boldsymbol{Q}$ in SSR is obtained by butterfly matrices.

Evaluation metrics: We evaluate the performance of all the compared algorithms in terms of approximation error, time cost, and test accuracy. The used kernel approximation measure here is the relative error in Frobenius form $\|\boldsymbol{K}-\hat{\boldsymbol{K}}\|_{\mathrm{F}} /\|\boldsymbol{K}\|_{\mathrm{F}}$ on a randomly selected subset with 1,000 observations. We record the time cost of each algorithm on generating feature mappings. Regarding to generalization performance, the classifier in this paper is chosen as the kernel ridge regression (KRR) with the closed-form formula $\left[\mathrm{SVGDB}^{+} 02, \mathrm{JSSS}^{+} 20\right]$. The regularization parameter $\lambda$ in KRR is tuned via 5 -fold inner cross validation over a grid of $\{0.0001,0.001,0.01,0.1,0.5,1,10\}$. Denote $\boldsymbol{\eta} \in \mathbb{R}^{D}$ as the coefficient vector in KRR, for classification, we use the sign function $\operatorname{sign}\left[\operatorname{Re}\left(\Psi(\boldsymbol{x})^{\top} \boldsymbol{\eta}\right)\right]$ to output a binary classification label. It can be extended to multi-class classification using the one-versus-one/all scheme. All experiments are repeated 10 trials.

\subsection{Evaluation for our deterministic rules}

Our deterministic rule generates the fixed-size feature mapping $\Phi(\boldsymbol{x}) \in \mathbb{R}^{D}$, e.g., $D=2 d+1$ in our thirddegree rule $(m=1)$ and $D=1+2 d^{2}$ in our fifth-degree rule $(m=2)$. In this case, we consider a deterministic setting, in which RFF, ORF, ROM, QMC and SSR are conducted under the same feature dimension $D$ for fair comparison.

The third-degree case: The generated feature mapping dimension is $D=2 d+1$ for all of compared algorithms. Figure 3 shows the mean of approximation error, the time cost, and the classification accuracy (with one standard deviation denoted by error bars) across the Gaussian kernel, where the blue bar indicates the third-degree case. We find that, our deterministic rule significantly decreases the approximation error of RFF, QMC, and SGQ, achieves a comparable performance with ORF and ROM, but is slightly inferior

\footnotetext{
${ }^{4}$ https://archive.ics.uci.edu/ml/datasets.html.
} 


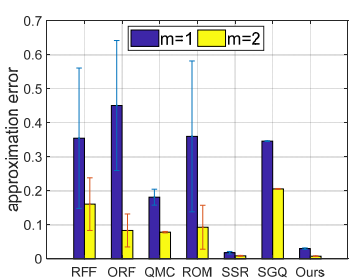

(a) $\mathrm{magicO}_{4}$
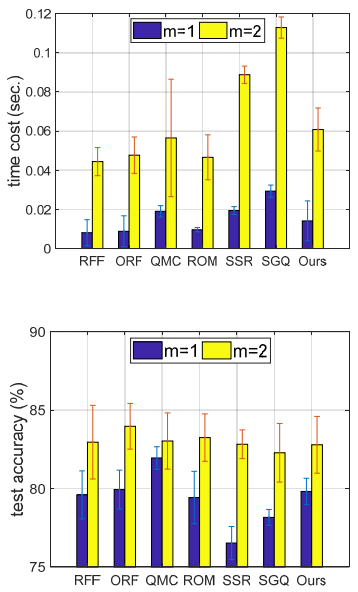

(i) $\mathrm{magic0}_{4}$

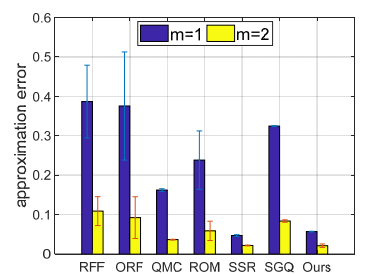

(b) letter
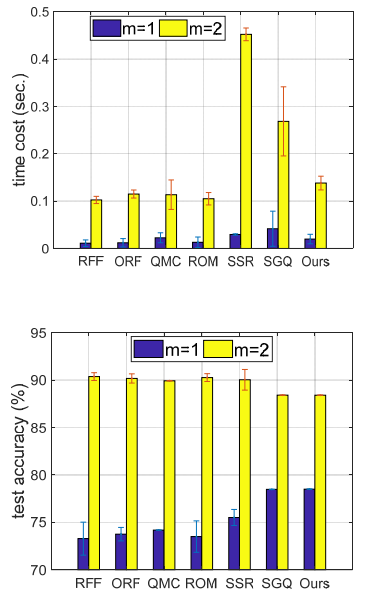

(j) letter

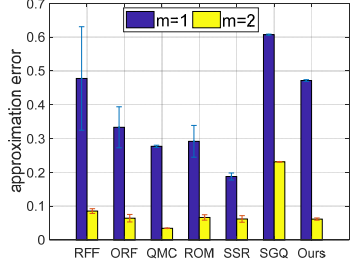

(c) ijcnn1
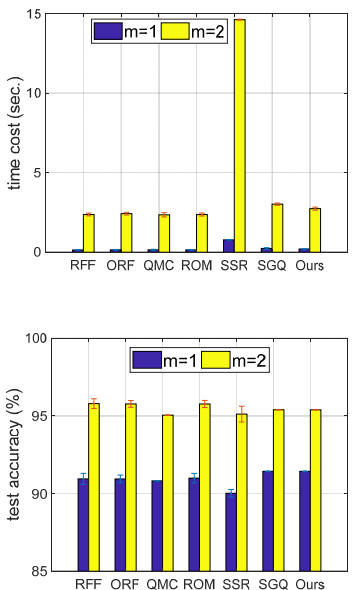

(k) ijcnn1

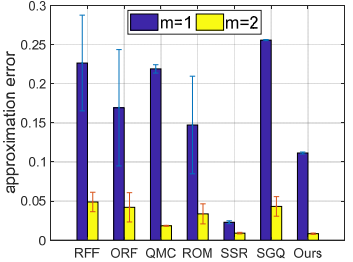

(d) covtype
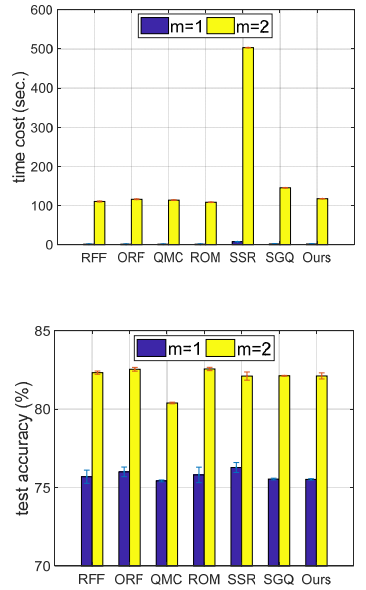

(l) covtype

Figure 4. Results on the first-order arc-cosine kernel in terms of approximation error (top), time cost (middle), and test accuracy (bottom).

to SSR. However, our deterministic rule obtains the similar time cost with RFF and is much efficient than SSR. In terms of generalization performance, most algorithms achieve the similar test accuracy on these datasets. Good kernel approximation quality indeed cannot guarantee the final good prediction. This is still an open question in theory, but, for the design of kernel approximation, it is reasonable to pursue small approximation errors. The reason may be that the approximated kernel is not necessarily optimal for classification/regression tasks, as discussed by $\left[\mathrm{AKM}^{+} 17\right.$, ZMDR19, LHCS20].

Figure 4 shows the related results across the first-order arc-cosine kernel. The trends of the compared algorithms is analogous to those across the Gaussian kernel in Figure 3. To be specific, our deterministic rule performs better than RFF, ORF, QMC, and SGQ in most cases but is narrowly inferior to SSR and ROM. Nevertheless, our method is as efficient as RFF to generate the feature mapping while SSR requires several times computational cost. That means, our rule achieves a good trade-off between the approximation quality and computational complexity. Regarding to test accuracy, all of the compared algorithms perform well on the ijcnn1 and covtype data sets but prohibit distinct results on the magic04 and letter data sets. Nevertheless, our empirical results demonstrate that our approach achieves comparable quality on the final tasks with other representative random features based algorithms. Especially worthy of mention is, the approximation error of each algorithm on the arc-cosine kernel is generally larger than that of Gaussian kernel. One reason is that the integrand $f$ in the Gaussian kernel corresponds to trigonometric functions that is infinitely differentiable; while $f$ in the first-order arc-cosine kernel is actually a ReLU function that is non-differentiable. In fact, the differentiable property on the integrand significantly affects the approximation performance in Monte Carlo sampling, QMC, and quadrature based algorithms.

The fifth-degree case: The generated feature mapping dimension is $D=2 d^{2}+1$ for all of compared algorithms except SGQ. Note that, the fifth-degree SGQ requires a higher dimension with $D=1+2 d^{2}+2 d$, see in Table 2, which is still taken into comparisons. Figures 3 and 4 shows results across the Gaussian kernel and the first-order arc-cosine kernel, respectively, as illustrated by the yellow bar. It can be noticed that, all of algorithms achieve significant improvements on the approximation quality and generalization 


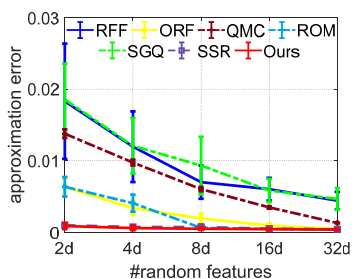

(a) magic04

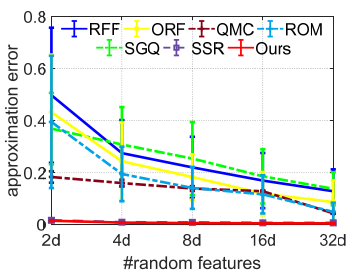

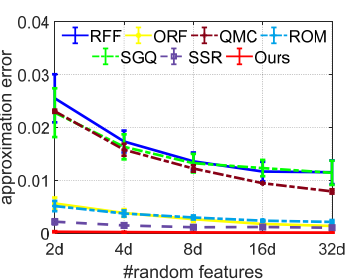

(b) letter

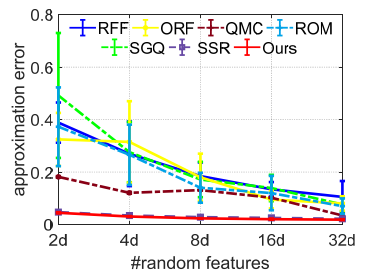

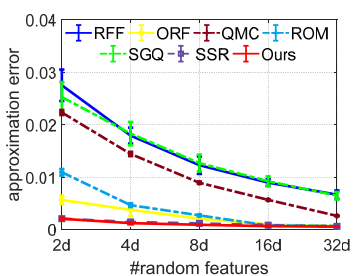

(c) ijcnn1

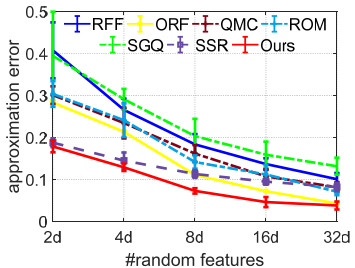

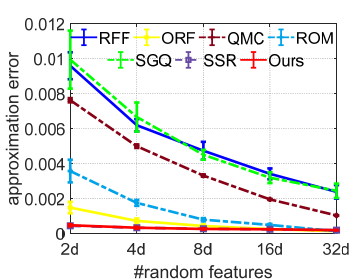

(d) covtype

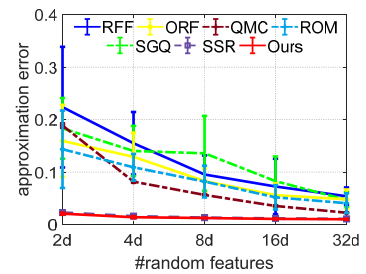

Figure 5. Kernel approximation error on the RBF kernel (top) and the first-order arc-cosine kernel (bottom).

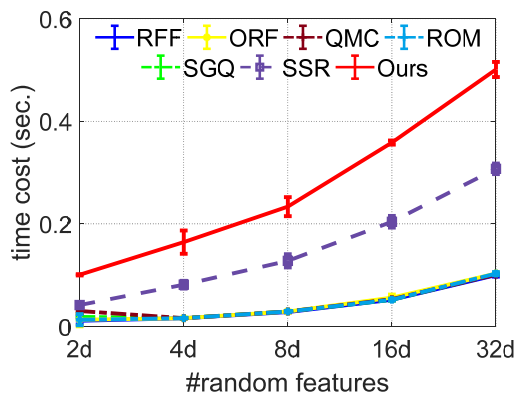

(a) magic04 $_{4}$

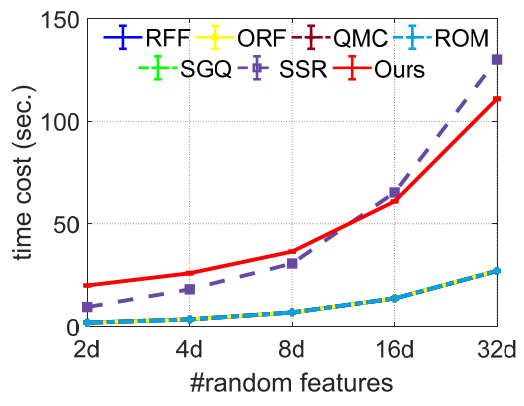

(b) covtype

Figure 6: Time cost across the Gaussian kernel.

performance when compared to the third-degree case. More importantly, our fifth-degree rule achieves the best kernel approximation performance and is still as efficient as RFF. Although SSR performs well, it is relatively time-consuming on the feature mapping.

Based on the above results, we conclude that our deterministic third/fifth-degree rules are quite efficient to achieve promising performance on the approximation quality.

\subsection{Evaluation for our stochastic rule}

Apart from the above setting for deterministic rules, we also evaluate our stochastic rule under a dimensionadaptive setting, in which the feature dimension works in the $D=\{2 d, 4 d, 8 d, 16 d, 32 d\}$ order followed by [MKBO18]. In fact, our deterministic third/fifth-degree rules are associated with $D=2 d$ and $D=2 d^{2}$ order, which covers the low/high dimensional case, respectively. Since the feature dimension $D$ in our fifth-degree rule is quite high, we only consider the stochastic third-degree rule in our experiment. To purse a better approximation performance, our stochastic third-degree rule in Eq. (4.5) obtain $\left\{\boldsymbol{\omega}_{i}\right\}_{i=1}^{D}$ by SSR rather than Monte Carlo sampling. To incorporate SSR into our stochastic rule, we directly use a random orthogonal matrix $\boldsymbol{Q}$ instead of butterfly matrices in our implementation. Regarding to SGQ, following with [DDSR17], we use subsampled grids to output a dimension-adaptive feature mapping.

Kernel approximation quality: Figure 5 shows the mean of approximation errors across the Gaussian kernel and the first-order arc-cosine kernel (with one standard deviation denoted by error bars) under different feature dimensionality with $D=\{2 d, 4 d, 8 d, 16 d, 32 d\}$. We find that our method achieves a promising approximation performance on these data sets when compared to these representative kernel approximation 


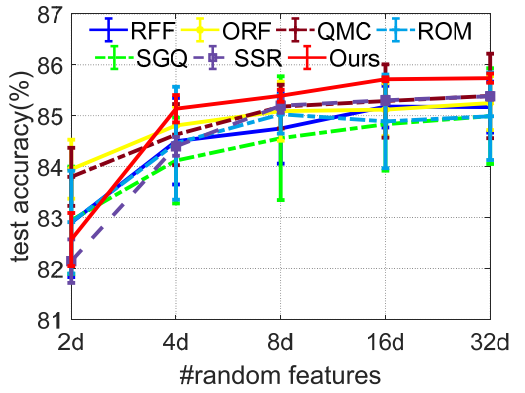

(a) $\operatorname{magic0} 4$

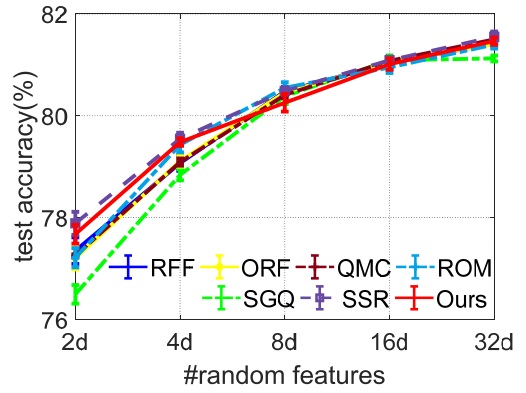

(b) covtype

Figure 7: Classification accuracy of all the compared algorithms across the Gaussian kernel.

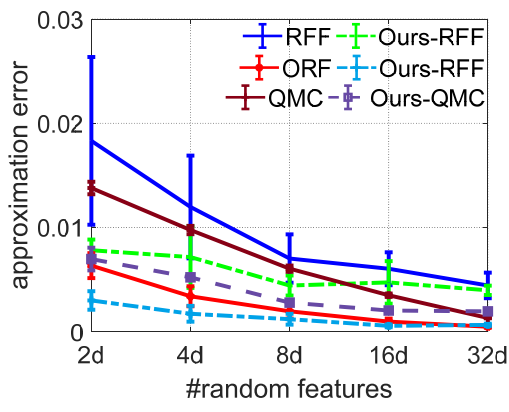

(a) approximation error

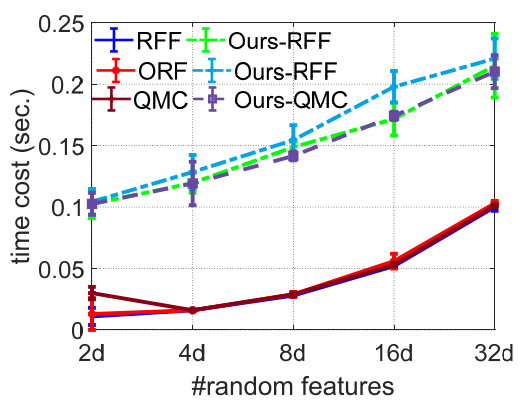

(b) time cost

Figure 8. Comparisons of various sampling schemes in Eq. (4.5) for $\left\{\boldsymbol{\omega}_{i}\right\}_{i=1}^{D}$ across the Gaussian kernel on the magic04 data set.

methods. Especially on the letter and ijcnn1 data sets, our stochastic rule outperforms SSR with a significant margin due to the applied control variate technique. Besides, SGQ [DDSR17] with subsampled grids performs narrowly to RFF as reported by their findings. Our results verify this phenomenon, i.e., SGQ with subsampled grids almost always coincide with RFF on these data sets, which demonstrates the superiority of our used control variate technique to some extent. Similarly, approximation results on the arc-cosine kernel are similar to that of Gaussian kernel approximation.

Time cost: Here we choose two data sets, magic04 and covtype, to evaluate the time cost for generating randomized feature maps. Results of all the compared algorithms across the Gaussian kernel are shown in Figure 6. We find that RFF, ORF, QMC, and SGQ with subsampled grids achieves similar time cost. While our stochastic rule equipped with SSR takes a little more time but still in the same order. Interestingly, in the high dimensional case (e.g., $D=32 d$ in covtype), our stochastic method takes less time than SSR. This is because, we directly use a random orthogonal matrix $\boldsymbol{Q}$ instead of butterfly matrices as the Fast Discrete Walsh-Hadamard Transform in SSR is actually not efficient in high dimensions.

Generalization performance: As mentioned before, the compared algorithms achieve the similar test accuracy under $D=2 d+1$ and $D=2 d^{2}+1$ regimes. Figure 7 shows the test accuracy of each algorithm across the Gaussian kernel on the magic04 and covtype data sets under varying feature dimensionality. As we expected, there is no distinct difference of these approaches on the final classification accuracy.

Various sampling schemes: In our stochastic rule, $\left\{\boldsymbol{\omega}_{i}\right\}_{i=1}^{D}$ in Eq. (4.5) is obtained by SSR instead of the Monte Carlo sampling for better approximation quality. Here we experimentally evaluate the performance of our stochastic rule equipped with various sampling schemes, i.e., $\left\{\boldsymbol{\omega}_{i}\right\}_{i=1}^{D}$ are obtained by RFF, ORF, and QMC, termed as "Ours-RFF", "Ours-ORF", and "Ours-QMC", respectively. Figure 8 shows the approximation error and time cost of the compared algorithms across the Gaussian kernel on the magic04 data set. It can be found that, using different sampling schemes, our stochastic rule always achieves significant variance reduction when compared to the original RFF, ORF, and QMC, which demonstrates the effectiveness of our used control variate technique in Eq. (4.5). Besides, we observe that, the variance reduction effect weaken or 
even disappear when $D$ is large. For example, "Ours-RFF" achieves the similar approximation performance with $\mathrm{RFF}$ when $D=32 d$. One reason might be that, the variance of our stochastic rule converges to that of $\mathrm{RFF}$ at a fast $\mathcal{O}\left(1 / d^{2}\right)$ rate, as demonstrated by Theorem 2.

Regarding to the time cost, our stochastic rules including "Ours-RFF", "Ours-ORF", and "Ours-QMC", take more time than RFF, ORF, and QMC due to the extra computational cost on the applied deterministic fully symmetric rule. However, the time complexity of these algorithms are still in the same order $\mathcal{O}(D d)$.

\section{Conclusion}

We present deterministic/stochastic quadrature methods based on the fully symmetric interpolatory rule to approximate the shift-invariant Gaussian kernel and rotation-invariant arc-cosine kernels. Specifically, the weighting function $p(\cdot)$ can be chosen as other fully symmetric distributions, e.g., student-t distribution in [GM98], which is able to expand the usage of considered kernels. Our deterministic method achieves a good trade-off between approximation quality and space/time efficiency; Our stochastic rule exhibits nice statistical properties and performs well on real datasets. By studying the relations between quadrature based algorithms, our unified framework demonstrate that, i) our deterministic interpolatory rule recovers SGQ when choosing a suitable choice of parameters; ii) SSR can be regarded as a doubly stochastic version of our deterministic rule via a random projection scheme and a randomized generator.

\section{Acknowledgement}

The research leading to these results has received funding from the European Research Council under the European Union's Horizon 2020 research and innovation program / ERC Advanced Grant E-DUALITY (787960). This paper reflects only the authors' views and the Union is not liable for any use that may be made of the contained information. This work was supported in part by Research Council KU Leuven: Optimization frameworks for deep kernel machines C14/18/068; Flemish Government: FWO projects: GOA4917N (Deep Restricted Kernel Machines: Methods and Foundations), PhD/Postdoc grant. This research received funding from the Flemish Government (AI Research Program). This work was supported in part by Ford KU Leuven Research Alliance Project KUL0076 (Stability analysis and performance improvement of deep reinforcement learning algorithms), EU H2020 ICT-48 Network TAILOR (Foundations of Trustworthy AI - Integrating Reasoning, Learning and Optimization), Leuven.AI Institute; and in part by the National Natural Science Foundation of China 61977046, in part by the National Key Research and Development Project (No. 2018AAA0100702), in part by National Science Foundation grants CCF-1657420 and CCF-1704828, and in part by SJTU Global Strategic Partnership Fund (2020 SJTU-CORNELL).

\section{References}

$\left[\mathrm{ADH}^{+} 19 \mathrm{a}\right]$ Sanjeev Arora, Simon Du, Wei Hu, Zhiyuan Li, and Ruosong Wang, Fine-grained analysis of optimization and generalization for overparameterized two-layer neural networks, Proceedings of International Conference on Machine Learning, 2019, pp. 322-332. 1

$\left[\mathrm{ADH}^{+} 19 \mathrm{~b}\right]$ Sanjeev Arora, Simon S. Du, Wei Hu, Zhiyuan Li, Russ R. Salakhutdinov, and Ruosong Wang, On exact computation with an infinitely wide neural net, Proceedings of Advances in Neural Information Processing Systems, 2019, pp. 8139-8148. 1

[AH09] Ienkaran Arasaratnam and Simon Haykin, Cubature kalman filters, IEEE Transactions on Automatic Control 54 (2009), no. 6, 1254-1269. 7

$\left[\mathrm{AKM}^{+} 17\right]$ Haim Avron, Michael Kapralov, Cameron Musco, Christopher Musco, Ameya Velingker, and Amir Zandieh, Random Fourier features for kernel ridge regression: Approximation bounds and statistical guarantees, Proceedings of the 34th International Conference on Machine Learning, 2017, pp. 253-262. 4, 14 
[ASYM16] Haim Avron, Vikas Sindhwani, Jiyan Yang, and Michael W. Mahoney, Quasi-Monte Carlo feature maps for shift-invariant kernels, Journal of Machine Learning Research 17 (2016), no. 1, 4096-4133. 4, 13

[Bac17] Francis Bach, On the equivalence between kernel quadrature rules and random feature expansions, Journal of Machine Learning Research 18 (2017), no. 1, 714-751. 4

[BLJO12] Francis Bach, Simon Lacoste-Julien, and Guillaume Obozinski, On the equivalence between herding and conditional gradient algorithms, Proceedings of the 29th International Coference on International Conference on Machine Learning, Omnipress, 2012, pp. 1355-1362. 4

[Boc05] Salomon Bochner, Harmonic analysis and the theory of probability, Courier Corporation, 2005. 23

$\left[\mathrm{BOC}^{+} 17\right] \quad$ François-Xavier Briol, Chris J Oates, Jon Cockayne, Wilson Ye Chen, and Mark Girolami, On the sampling problem for kernel quadrature, Proceedings of the 34th International Conference on Machine Learning, JMLR.org, 2017, pp. 586-595. 4

[Coo97] Ronald Cools, Constructing cubature formulae: the science behind the art, Acta Numerica 6 (1997), 1-54. 4

[CRCW19] Krzysztof Choromanski, Mark Rowland, Wenyu Chen, and Adrian Weller, Unifying orthogonal Monte Carlo methods, Proceedings of International Conference on Machine Learning, 2019, pp. 1203-1212. 4, 9

[CRW17] Krzysztof M. Choromanski, Mark Rowland, and Adrian Weller, The unreasonable effectiveness of structured random orthogonal embeddings, Proceedings of Advances in Neural Information Processing Systems, 2017, pp. 219-228. 1, 4, 13

[CS09] Youngmin Cho and Lawrence K Saul, Kernel methods for deep learning, Advances in Neural Information Processing Systems, 2009, pp. 342-350. 12

[Dan17] Amit Daniely, SGD learns the conjugate kernel class of the network, Proceedings of Advances in Neural Information Processing Systems, 2017, pp. 2422-2430. 1

[DDSR17] Tri Dao, Christopher M. De Sa, and Christopher Ré, Gaussian quadrature for kernel features, Proceedings of Advances in neural information processing systems, 2017, pp. 6107-6117. 1, 3, $4,6,7,9,13,15,16$

[DLG $\left.{ }^{+} 20\right]$ Zhiyuan Dang, Xiang Li, Bin Gu, Cheng Deng, and Heng Huang, Large-scale nonlinear AUC Maximization via triply stochastic gradients, IEEE Transactions on Pattern Analysis and Machine Intelligence (2020), 1-14. 1

[dRBK20] Stéphane d'Ascoli, Maria Refinetti, Giulio Biroli, and Florent Krzakala, Double trouble in double descent: Bias and variance(s) in the lazy regime, arXiv preprint arXiv:2003.01054 (2020). 1

[DW18] Edgar Dobriban and Stefan Wager, High-dimensional asymptotics of prediction: Ridge regression and classification, Annals of Statistics 46 (2018), no. 1, 247-279. 9, 26

[EK10] Noureddine El Karoui, The spectrum of kernel random matrices, Annals of Statistics 38 (2010), no. $1,1-50.9,12,26$

[EMM20] Tamás Erdélyi, Cameron Musco, and Christopher Musco, Fourier sparse leverage scores and approximate kernel learning, Proceedings of Advances in Neural Information Processing Systems, 2020, pp. 1-10. 4

[Eva9] Gwynne Evans, Practical numerical integration, Wiley New York, 1993. 4 
[Gar12] Jochen Garcke, Sparse grids in a nutshell, Sparse grids and applications, Springer, 2012, pp. $57-80.7$

[GK96] Alan Genz and Bradley D Keister, Fully symmetric interpolatory rules for multiple integrals over infinite regions with gaussian weight, Journal of Computational and Applied Mathematics 71 (1996), no. 2, 299-309. 3, 4, 5, 6

[GM98] Alan Genz and John Monahan, Stochastic integration rules for infinite regions, SIAM Journal on Scientific Computing 19 (1998), no. 2, 426-439. 4, 5, 7, 10, 17

[GS18] Bertrand Gauthier and Johan A.K. Suykens, Optimal quadrature-sparsification for integral operator approximation, SIAM Journal on Scientific Computing 40 (2018), no. 5, A3636A3674. 4

[HN07] Aicke Hinrichs and Erich Novak, Cubature formulas for symmetric measures in higher dimensions with few points, Mathematics of computation 76 (2007), no. 259, 1357-1372. 7, 10

[HS79] Joseph Hammer and Jennifer Seberry, Higher-dimensional orthogonal designs and Hadamard matrices II, Proceedings of the 9th Manitoba Conference on Numerical Mathematics, 1979, pp. 23-29. 11

[HW08] Florian Heiss and Viktor Winschel, Likelihood approximation by numerical integration on sparse grids, Journal of Econometrics 144 (2008), no. 1, 62-80. 2, 4, 9

[JŞS $\left.{ }^{+} 20\right] \quad$ Arthur Jacot, Berfin Şimşek, Francesco Spadaro, Clément Hongler, and Franck Gabriel, Kernel alignment risk estimator: Risk prediction from training data, Proceedings of Advances in Neural Information Processing Systems, 2020, pp. 1-9. 12, 13

[JXC15] Bin Jia, Ming Xin, and Yang Cheng, Relations between sparse-grid quadrature rule and spherical-radial cubature rule in nonlinear Gaussian estimation, IEEE Transactions on Automatic Control 60 (2015), no. 1, 199-204. 26

[KE19] Mehran Kafai and Kave Eshghi, CROification: accurate kernel classification with the efficiency of sparse linear SVM, IEEE Transactions on Pattern Analysis and Machine Intelligence $4 \mathbf{1}$ (2019), no. 1, 34-48. 1

[KS18] Toni Karvonen and Simo Sarkka, Fully symmetric kernel quadrature, SIAM Journal on Scientific Computing 40 (2018), no. 2, A697-A720. 4, 5, 11

$\left[\mathrm{LHC}^{+} 20\right] \quad$ Fanghui Liu, Xiaolin Huang, Yudong Chen, Jie Yang, and Johan A.K. Suykens, Random Fourier features via fast surrogate leverage weighted sampling, Proceedings of Thirty-Fourth AAAI Conference on Artificial Intelligence, 2020, pp. 4844-4851. 4

[LHCS20] Fanghui Liu, Xiaolin Huang, Yudong Chen, and Johan A.K. Suykens, Random features for kernel approximation: A survey in algorithms, theory, and beyond, arXiv preprint arXiv:2004.11154 (2020). 3, 14

$\left[\mathrm{LHG}^{+} 20\right] \quad$ Fanghui Liu, Xiaolin Huang, Chen Gong, Jie Yang, and Li Li, Learning data-adaptive nonparametric kernels, Journal of Machine Learning Research 21 (2020), no. 208, 1-39. 1

[LPSS ${ }^{+}$14] David Lopez-Paz, Suvrit Sra, Alex J. Smola, Zoubin Ghahramani, and Bernhard Schölkopf, Randomized nonlinear component analysis, Proceedings of the International Conference on Machine Learning, 2014, pp. 1359-1367. 1

[LR20] Tengyuan Liang and Alexander Rakhlin, Just interpolate: Kernel "ridgeless" regression can generalize, Annals of Statistics 48 (2020), no. 3, 1329-1347. 9, 26

[Lyu17] Yueming Lyu, Spherical structured feature maps for kernel approximation, Proceedings of the 34th International Conference on Machine Learning, JMLR.org, 2017, pp. 2256-2264. 2, 4 
[MKBO18] Marina Munkhoeva, Yermek Kapushev, Evgeny Burnaev, and Ivan Oseledets, Quadraturebased features for kernel approximation, Proceedings of Advances in Neural Information Processing Systems, 2018, pp. 9147-9156. 1, 2, 3, 4, 6, 7, 9, 10, 11, 12, 13, 15

[MS67] J McNamee and Frank Stenger, Construction of fully symmetric numerical integration formulas of fully symmetric numerical integration formulas, Numerische Mathematik 10 (1967), no. $4,327-344.4$

[Nie92] Harald Niederreiter, Random number generation and quasi-monte carlo methods, vol. 63, SIAM, 1992. 4

[NR99] Erich Novak and Klaus Ritter, Simple cubature formulas with high polynomial exactness, Constructive approximation 15 (1999), no. 4, 499-522. 3

[OG19] Dino Oglic and Thomas Gärtner, Scalable learning in reproducing kernel kreŭn spaces, Proceedings of International Conference on Machine Learning, 2019, pp. 4912-4921. 1

[PBV18] Edouard Pauwels, Francis Bach, and Jean-Philippe Vert, Relating leverage scores and density using regularized christoffel functions, Proceedings of Advances in Neural Information Processing Systems, 2018, pp. 1663-1672. 4

[RCCR18] Alessandro Rudi, Daniele Calandriello, Luigi Carratino, and Lorenzo Rosasco, On fast leverage score sampling and optimal learning, Proceedings of Advances in Neural Information Processing Systems, 2018, pp. 5672-5682. 4

[RM85] Reuven Y Rubinstein and Ruth Marcus, Efficiency of multivariate control variates in monte carlo simulation, Operations Research 33 (1985), no. 3, 661-677. 8

[RR07] Ali Rahimi and Benjamin Recht, Random features for large-scale kernel machines, Proceedings of Advances in Neural Information Processing Systems, 2007, pp. 1177-1184. 1, 2, 13

[SGT18] Yitong Sun, Anna Gilbert, and Ambuj Tewari, But how does it work in theory? Linear SVM with random features, Proceedings of Advances in Neural Information Processing Systems, 2018, pp. 3383-3392. 1

[SS03] Bernhard Schölkopf and Alexander J. Smola, Learning with kernels: support vector machines, regularization, optimization, and beyond, MIT Press, 2003. 1

[SVGDB ${ }^{+}$02] Johan A.K. Suykens, Tony Van Gestel, Jos De Brabanter, Bart De Moor, and Joos Vandewalle, Least squares support vector machines, World Scientific, 2002. 1, 13

[YSAM14] Jiyan Yang, Vikas Sindhwani, Haim Avron, and Michael Mahoney, Quasi-Monte Carlo feature maps for shift-invariant kernels, Proceedings of the International Conference on Machine Learning, 2014, pp. 485-493. 1

[YSC $\left.{ }^{+} 16\right] \quad$ Felix Xinnan Yu, Ananda Theertha Suresh, Krzysztof Choromanski, Daniel Holtmannrice, and Sanjiv Kumar, Orthogonal random features, Proceedings of Advances in Neural Information Processing Systems, 2016, pp. 1975-1983. 1, 4, 9, 13, 24, 26

[ZMDR19] Jian Zhang, Avner May, Tri Dao, and Christopher Re, Low-precision random Fourier features for memory-constrained kernel approximation, Proceedings of the 22nd International Conference on Artificial Intelligence and Statistics, 2019, pp. 1264-1274. 14 


\section{A Fifth-degree Fully Symmetric Interpolatory Rules}

When choosing $m=2$ in Eq. (3.1), we obtain a fifth-degree rule $Q^{(2, d)}$ with $\|\boldsymbol{p}\|_{1} \leq 2$ to further improve the approximation accuracy. To derive the fifth-degree rule, we can cast it in three cases, i.e., $\|\boldsymbol{p}\|_{1}=0$, $\|\boldsymbol{p}\|_{1}=1$, and $\|\boldsymbol{p}\|_{1}=2$.

If $\|\boldsymbol{p}\|_{1}=0$, we have $p_{i}=0, \boldsymbol{\lambda}=\mathbf{0}$, and $K=0$. Then the weight $a_{0}^{(2, d)}$ is

$$
a_{0}^{(2, d)}=\sum_{\|\boldsymbol{u}\|_{1} \leq 2} \prod_{i=1}^{d} \frac{b_{u_{i}}}{\prod_{j=0, \neq 0}^{u_{i}}\left(\lambda_{0}^{2}-\lambda_{j}^{2}\right)}=1-\frac{d}{\lambda_{1}^{2}}+\frac{d(d-1)}{2 \lambda_{1}^{4}}+\frac{d\left(3-\lambda_{1}^{2}\right)}{\lambda_{1}^{2} \lambda_{2}^{2}},
$$

where $b_{2}=3-\lambda_{1}^{2}$ is obtained by Eq. (2.3). In our derivation, $\|\boldsymbol{u}\|_{1} \leq 2$ is cast into three cases: $\boldsymbol{u}=\mathbf{0}$, $\|\boldsymbol{u}\|_{1}=1$, and $\|\boldsymbol{u}\|_{1}=2$ for calculation.

If $\|\boldsymbol{p}\|_{1}=1$, only one element of $\boldsymbol{p}$ is 1 and the remaining are zero. We thereby have $K=1$ and $\boldsymbol{\lambda}=\lambda_{1} \boldsymbol{e}_{i}$ with $i=1,2, \ldots, d$, where $\boldsymbol{e}_{i}$ is a unit vector with the $i$-th element being 1 . Without loss of generality, assuming $\boldsymbol{p}=[1,0, \cdots, 0]$, the weight $a_{1}^{(2, d)}$ is computed as

$$
a_{1}^{(2, d)}=\frac{1}{2} \sum_{\|\boldsymbol{u}\|_{1} \leq 1} \prod_{i=1}^{d} \frac{b_{u_{i}+p_{i}}}{\prod_{j=0, \neq p_{i}}^{u_{i}+p_{i}}\left(\lambda_{p_{i}}^{2}-\lambda_{j}^{2}\right)}=\frac{1}{2 \lambda_{1}^{2}}+\frac{3-\lambda_{1}^{2}}{2 \lambda_{1}^{2}\left(\lambda_{1}^{2}-\lambda_{2}^{2}\right)}-\frac{d-1}{2 \lambda_{1}^{4}},
$$

where $\|\boldsymbol{u}\|_{1} \leq 1$ is cast into two cases: $\boldsymbol{u}=\mathbf{0}$ and $\|\boldsymbol{u}\|_{1}=1$ for derivation.

If $\|\boldsymbol{p}\|_{1}=2$, the derivation is a little complex and we cast it into two cases. One is that there are two elements in $\boldsymbol{p}$ being 1 , i.e., $p_{i}=p_{j}=1$ with $i \neq j$. The other is only one element of $\boldsymbol{p}$ being 2 , i.e., $p_{i}=2$. For the $p_{i}=p_{j}=1$ case, we have $K=2$ and $\boldsymbol{\lambda}=\lambda_{1} \boldsymbol{s}_{l}^{+}$or $\boldsymbol{\lambda}=\lambda_{1} \boldsymbol{s}_{l}^{-}$, where the point sets of $\boldsymbol{s}_{l}^{+}$and $\boldsymbol{s}_{l}^{-}$are given by

$$
\begin{aligned}
& \left\{\boldsymbol{s}_{l}^{+}\right\}_{l=1}^{d(d-1) / 2}:=\left\{\boldsymbol{e}_{i}+\boldsymbol{e}_{j}: i<j, i, j=1,2, \cdots, d\right\} \\
& \left\{\boldsymbol{s}_{l}^{-}\right\}_{l=1}^{d(d-1) / 2}:=\left\{\boldsymbol{e}_{i}-\boldsymbol{e}_{j}: i<j, i, j=1,2, \cdots, d\right\}
\end{aligned}
$$

Without loss of generality, assuming $\boldsymbol{p}=[1,1,0, \cdots, 0]$, the weight $a_{2}^{(2, d)}$ is

$$
a_{2}^{(2, d)}=\frac{1}{4} \prod_{i=1}^{d} \frac{b_{p_{i}}}{\prod_{j=0, \neq p_{i}}^{p_{i}}\left(\lambda_{p_{i}}^{2}-\lambda_{j}^{2}\right)}=\frac{1}{4}\left[\frac{b_{1}}{\left(\lambda_{1}^{2}-\lambda_{0}^{2}\right)}\right]^{2}=\frac{1}{4 \lambda_{1}^{4}} .
$$

For the $p_{i}=2$ case, we have $K=1$ and $\boldsymbol{\lambda}=\lambda_{2} \boldsymbol{e}_{i}$. Without loss of generality, assuming $\boldsymbol{p}=[2,0, \cdots, 0]$, the weight $a_{3}^{(2, d)}$ is computed as

$$
a_{3}^{(2, d)}=\frac{1}{2} \prod_{i=1}^{d} \frac{b_{p_{i}}}{\prod_{j=0, \neq p_{i}}^{p_{i}}\left(\lambda_{p_{i}}^{2}-\lambda_{j}^{2}\right)}=\frac{3-\lambda_{1}^{2}}{2 \lambda_{2}^{2}\left(\lambda_{2}^{2}-\lambda_{1}^{2}\right)} .
$$

Accordingly, combining the derived weights in Eqs. (A.1), (A.2), (A.3), and (A.4), the fifth-degree full symmetric interpolatory rule is

$$
\begin{aligned}
Q^{(2, d)}(f) & =a_{0}^{(2, d)} f(\mathbf{0})+a_{1}^{(2, d)} \sum_{i=1}^{d}\left[f\left(\lambda_{1} \boldsymbol{e}_{i}\right)+f\left(-\lambda_{1} \boldsymbol{e}_{i}\right)\right] \\
& +a_{2}^{(2, d)} \sum_{i=1}^{d(d-1) / 2}\left[f\left(\lambda_{1} \boldsymbol{s}_{i}^{+}\right)+f\left(-\lambda_{1} \boldsymbol{s}_{i}^{+}\right)+f\left(\lambda_{1} \boldsymbol{s}_{i}^{-}\right)+f\left(-\lambda_{1} \boldsymbol{s}_{i}^{-}\right)\right]+a_{3}^{(2, d)} \sum_{i=1}^{d}\left[f\left(\lambda_{2} \boldsymbol{e}_{i}\right)+f\left(-\lambda_{2} \boldsymbol{e}_{i}\right)\right] \\
& :=a_{0}^{(2, d)} f(\mathbf{0})+\sum_{j=1}^{2 d}\left(a_{1}^{(2, d)} f\left(\boldsymbol{P}_{j, 1}\right)+a_{3}^{(2, d)} f\left(\boldsymbol{P}_{j, 3}\right)\right)+a_{2}^{(2, d)} \sum_{j=1}^{2 d(d-1)} f\left(\boldsymbol{P}_{j, 2}\right)
\end{aligned}
$$

where

$$
\boldsymbol{P}_{j, 1}=\left\{\begin{array}{c}
\lambda_{1} \boldsymbol{e}_{i} ; a_{i}=\frac{1}{2 d} ; 1 \leq i \leq d \\
-\lambda_{1} \boldsymbol{e}_{i-d} ; d+1 \leq i \leq 2 d
\end{array}\right.
$$




$$
\begin{gathered}
\boldsymbol{P}_{j, 3}=\left\{\begin{array}{c}
\lambda_{2} \boldsymbol{e}_{i} ; 1 \leq i \leq d \\
-\lambda_{2} \boldsymbol{e}_{i-d} ; d+1 \leq i \leq 2 d
\end{array}\right. \\
\boldsymbol{P}_{j, 2}= \begin{cases}\lambda_{1}\left(\boldsymbol{e}_{i}+\boldsymbol{e}_{t}\right) & i, t=1, \ldots, d ; i<t \\
\lambda_{1}\left(\boldsymbol{e}_{i}-\boldsymbol{e}_{t}\right) & i, t=1, \ldots, d ; i<t \\
\lambda_{1}\left(-\boldsymbol{e}_{i}+\boldsymbol{e}_{t}\right) & i, t=1, \ldots, d ; i<t \\
\lambda_{1}\left(-\boldsymbol{e}_{i}-\boldsymbol{e}_{t}\right) & i, t=1, \ldots, d ; i<t\end{cases}
\end{gathered}
$$

Similar to the third-degree rule, we also choose $\lambda_{i}$ by successive extensions of the one-dimensional 3-point Gauss-Hermite rule.

\section{B Statistical Guarantees of Stochastic Interpolatory Rules}

This section includes three parts:

- in Section B.1, we prove Theorem 1, that is, our third-degree stochastic interpolatory rule $R_{m}(f)$ is an unbiased $2 m+1$ degree rule for $I_{d}(f)$ with $m=1$.

- in Section B.2, we prove Theorem 2 that gives the variance of our third-degree stochastic interpolatory rule, i.e., $\mathbb{V}\left[R_{1}(f)\right]$.

\section{B.1 Proof of Theorem 1}

Proof Here we prove the unbiased property of the third-degree rule, and further, under suitable modifications, our proofs can be extended to that of the fifth-degree stochastic rule.

We compute $I\left(\tilde{a}_{\boldsymbol{p}}^{(1, d)}(\boldsymbol{\omega})\right)$ as follows. For $\tilde{a}_{0}^{(1, d)}$

$$
I\left(\tilde{a}_{0}^{(1, d)}\right)=\int_{\mathbb{R}^{d}} p(\boldsymbol{\omega})\left(1-\frac{\sum_{i=1}^{d} \omega_{i}^{2}}{\lambda_{1}^{2}}\right) \mathrm{d} \boldsymbol{\omega}=a_{0}^{(1, d)} .
$$

For $\tilde{a}_{1}^{(1, d)}$, we have

$$
I\left(\tilde{a}_{1}^{(1, d)}\right)=\frac{1}{2 d \lambda_{1}^{2}} \int_{\mathbb{R}^{d}} p(\boldsymbol{\omega})\left(\sum_{i=1}^{d} \omega_{i}^{2}\right) \mathrm{d} \boldsymbol{\omega}=a_{1}^{(1, d)} .
$$

So we have $a_{\boldsymbol{p}}^{(1, d)}=I_{d}\left[\tilde{a}_{\boldsymbol{p}}^{(1, d)}(\boldsymbol{\omega})\right]$, and thus $Q^{(1, d)}(f)=I_{d}\left[M^{(1, d)}(f, \boldsymbol{\omega})\right]$. Due to $I(f)=\mathbb{E}_{\boldsymbol{\omega} \sim p(\cdot)}[f(\boldsymbol{\omega})]$, we have $Q^{(1, d)}(f)=\mathbb{E}_{\boldsymbol{\omega} \sim p(\cdot)}\left[M^{(1, d)}(f, \boldsymbol{\omega})\right]$. Based on this, the expectation of $r_{1}(f, \boldsymbol{\omega})$ is

$$
\begin{aligned}
\mathbb{E}_{\boldsymbol{\omega}}\left[r_{1}(f, \boldsymbol{\omega})\right] & =\mathbb{E}[f(\boldsymbol{\omega})]-\mathbb{E}\left[M^{(1, d)}(f, \boldsymbol{\omega})\right]+\mathbb{E}\left\{I_{d}\left[M^{(1, d)}(f)\right]\right\} \\
& =I_{d}[f(\boldsymbol{\omega})]-Q^{(1, d)}(f)+Q^{(1, d)}(f) \\
& =I_{d}(f) .
\end{aligned}
$$

Accordingly, if $D$ random nodes $\left\{\boldsymbol{\omega}_{i}\right\}_{i=1}^{D}$ are sampling from $p(\boldsymbol{\omega})$, the average $R_{1}(f)$ is unbiased for $I_{d}(f)$.

Besides, if we choose $f(\boldsymbol{\omega})=\boldsymbol{\omega}^{2 \boldsymbol{u}}$ with $\|\boldsymbol{u}\|_{1} \leq 1$, then we have $r_{1}(f, \boldsymbol{\omega})=Q^{(1, d)}(f)=I_{d}\left[M^{(1, d)}(f, \boldsymbol{\omega})\right]$. If we choose $f(\boldsymbol{\omega})=\boldsymbol{\omega}^{\boldsymbol{u}}$ in which at least one element of $\boldsymbol{u}$ is odd, we have $r_{1}(f, \boldsymbol{\omega})=0$. So it means that $r_{1}(f, \boldsymbol{\omega})$ is a third-degree rule for $I_{d}(f)$. Hence, $R_{1}(f)$ is an unbiased third-degree stochastic rule for $I_{d}(f)$, which concludes the proof.

\section{B.2 Proof of Theorem 2 and its condition verification}

This section aims to prove Theorem 2 including three parts. In Section B.2.1, we present Lemma B.1 that is used to prove Theorem 2. The proof of Theorem 2 can be found in Section B.2.2. In Section B.2.3, we verify the condition for $\mathbb{V}\left[R_{1}(f)\right]-\mathbb{V}[\mathrm{RFF}]<0$ in Theorem 2 holds for most cases. 


\section{B.2.1 Proof of Lemma B.1}

To aid the proof of Theorem 2, we need the following lemma.

Lemma B.1. Denote $\boldsymbol{\omega}=\left[\omega_{1}, \omega_{2}, \cdots, \omega_{d}\right]^{\top} \sim \mathcal{N}\left(\mathbf{0}, \boldsymbol{I}_{d}\right), \boldsymbol{z}=\boldsymbol{x}-\boldsymbol{y} / \sigma=\left[z_{1}, z_{2}, \cdots, z_{d}\right]^{\top}$, and $f(\boldsymbol{\omega})=$ $\cos \left(\boldsymbol{\omega}^{\top} \boldsymbol{z}\right)$, we have

$$
\mathbb{E}_{\boldsymbol{\omega}}\left(f(\boldsymbol{\omega}) \sum_{j=1}^{d} \omega_{j}^{2}\right)=e^{-\frac{\|\boldsymbol{z}\|_{2}^{2}}{2}}\left(d-\|\boldsymbol{z}\|_{2}^{2}\right) .
$$

Proof We expand $\mathbb{E}_{\boldsymbol{\omega}}\left(f(\boldsymbol{\omega}) \sum_{j=1}^{d} \omega_{j}^{2}\right)$ as

$$
\mathbb{E}_{\boldsymbol{\omega}}\left(f(\boldsymbol{\omega}) \sum_{j=1}^{d} \omega_{j}^{2}\right)=\sum_{j=1}^{d} \mathbb{E}_{\boldsymbol{\omega}}\left[\omega_{j}^{2} \cos \left(\boldsymbol{\omega}^{\top} \boldsymbol{z}\right)\right] .
$$

The $j$-th term $\omega_{j}^{2} \cos \left(\boldsymbol{\omega}^{\top} \boldsymbol{z}\right)$ can be reformulated as

$$
\begin{aligned}
\omega_{j}^{2} \cos \left(\boldsymbol{\omega}^{\top} \boldsymbol{z}\right) & =\omega_{j}^{2} \cos \left(\omega_{j} z_{j}+\sum_{t=1, \neq j}^{d} \omega_{t} z_{t}\right) \\
& =\omega_{j}^{2}\left[\cos \left(\omega_{j} z_{j}\right) \cos \left(\sum_{t=1, \neq j}^{d} \omega_{t} z_{t}\right)-\sin \left(\omega_{j} z_{j}\right) \sin \left(\sum_{t=1, \neq j}^{d} \omega_{t} z_{t}\right)\right] .
\end{aligned}
$$

Now we compute $\mathbb{E}_{\omega_{j}}\left[\omega_{j}^{2} \cos \left(\omega_{j} z_{j}\right)\right]$ as follows.

$$
\begin{aligned}
\mathbb{E}\left[\omega_{j}^{2} \cos \left(\omega_{j} z_{j}\right)\right] & =\frac{1}{\sqrt{2 \pi}} \int_{-\infty}^{\infty} \omega_{j}^{2} \cos \left(\omega_{j} z_{j}\right) e^{-\frac{\omega_{j}^{\top} \omega_{j}}{2}} \mathrm{~d} \omega_{j} \\
& =\operatorname{Re}\left(\int_{-\infty}^{\infty} \frac{1}{\sqrt{2 \pi}} \omega_{j}^{2} e^{\mathrm{i} \omega_{j} z_{j}} e^{-\frac{\omega_{j}^{\top} \omega_{j}}{2}} \mathrm{~d} \omega_{j}\right) \\
& =\operatorname{Re}\left(\int_{-\infty}^{\infty} \frac{1}{\sqrt{2 \pi}} \omega_{j}^{2} e^{-\frac{z_{j}^{2}}{2}} e^{-\frac{\left(\omega_{j}-\mathrm{i} z_{j}\right)^{2}}{2}} \mathrm{~d} \omega_{j}\right) \\
& =e^{-\frac{z_{j}^{2}}{2}} \mathbb{E}_{\omega_{j}}\left(\omega_{j}^{2}\right) \text { with } \omega_{j} \sim \mathcal{N}\left(\mathrm{i} z_{j}, 1\right) \\
& =e^{-\frac{z_{j}^{2}}{2}}\left[1+\left(\mathrm{i} z_{j}\right)^{2}\right]=e^{-\frac{z_{j}^{2}}{2}}\left(1-z_{j}^{2}\right),
\end{aligned}
$$

where i denotes the imaginary unit. Similarly, $\mathbb{E}\left[\omega_{j}^{2} \sin \left(\omega_{j} z_{j}\right)\right]$ can be computed as

$$
\mathbb{E}\left[\omega_{j}^{2} \sin \left(\omega_{j} z_{j}\right)\right]=\operatorname{Im}\left(\int_{-\infty}^{\infty} \frac{\omega_{j}^{2} e^{-\frac{z_{j}^{2}}{2}}}{\sqrt{2 \pi}} e^{-\frac{\left(\omega_{j}-\mathrm{i} z_{j}\right)^{2}}{2}} \mathrm{~d} \omega_{j}\right)=0 .
$$

Besides, by virtue of the above derivation, or directly using Bochner theorem [Boc05] for the Gaussian kernel $\mathbb{E}_{\boldsymbol{\omega}}\left[\cos \left(\boldsymbol{\omega}^{\top} \boldsymbol{z}\right)\right]=e^{-\|\boldsymbol{z}\|_{2}^{2} / 2}$, we have

$$
\mathbb{E}_{\left\{\omega_{1}, \cdots, \omega_{d}\right\} \backslash \omega_{j}}\left[\cos \left(\sum_{t=1, \neq j}^{d} \omega_{t} z_{t}\right)\right]=e^{-\frac{\|z\|_{2}^{2}-z_{j}^{2}}{2}} .
$$

Combine the above equations in Eqs. (B.1), (B.2), (B.3), and (B.4), we have

$$
\begin{aligned}
\mathbb{E}_{\boldsymbol{\omega}}\left[\omega_{j}^{2} \cos \left(\boldsymbol{\omega}^{\top} \boldsymbol{z}\right)\right] & =\mathbb{E}_{\omega_{j}}\left[\omega_{j}^{2} \cos \left(\omega_{j} z_{j}\right)\right] \mathbb{E}_{\left\{\omega_{1}, \cdots, \omega_{d}\right\} \backslash \omega_{j}}\left[\cos \left(\sum_{t=1, \neq j}^{d} \omega_{t} z_{t}\right)\right] \\
& =e^{-\frac{z_{j}^{2}}{2}}\left(1-z_{j}^{2}\right) e^{-\frac{\|z\|_{2}^{2}-z_{j}^{2}}{2}} \\
& =\left(1-z_{j}^{2}\right) e^{-\frac{\|\boldsymbol{z}\|_{2}^{2}}{2}} .
\end{aligned}
$$


Accordingly, we can conclude

$$
\mathbb{E}_{\boldsymbol{\omega}}\left(f(\boldsymbol{\omega}) \sum_{j=1}^{d} \omega_{j}^{2}\right)=\sum_{j=1}^{d} \mathbb{E}_{\boldsymbol{\omega}}\left[\omega_{j}^{2} \cos \left(\boldsymbol{\omega}^{\top} \boldsymbol{z}\right)\right]=e^{-\frac{\|\boldsymbol{z}\|_{2}^{2}}{2}} \sum_{i=1}^{d}\left(1-z_{j}^{2}\right),
$$

which yields the final result.

\section{B.2.2 Proof of Theorem 2}

In the next, we are ready to prove Theorem 2.

Proof For ease of description, we use some short notations including $\sum_{i=1}^{d}[f]:=\sum_{i=1}^{d}\left[f\left(\lambda_{1} \boldsymbol{e}_{i}\right)+f\left(-\lambda_{1} \boldsymbol{e}_{i}\right)\right]$, $Q:=Q^{(1, d)}(f)$, and $z:=\|\boldsymbol{z}\|_{2}$.

Recall RFF, its kernel approximation form is obtained via the Monte Carlo sampling

$$
\frac{1}{D} \sum_{i=1}^{D} \cos \left(\boldsymbol{\omega}_{i}^{\top} \boldsymbol{z}\right), \quad \boldsymbol{\omega}_{i} \sim \mathcal{N}\left(\mathbf{0}, \boldsymbol{I}_{d}\right)
$$

According to $\mathbb{E}\left[\cos \left(\boldsymbol{\omega}^{\top} \boldsymbol{z}\right)\right]=e^{-z^{2} / 2}$ and $\mathbb{V}\left[\cos \left(\boldsymbol{\omega}^{\top} \boldsymbol{z}\right)\right]=\left(1-e^{-z^{2}}\right)^{2} / 2\left[\mathrm{YSC}^{+} 16\right]$, we have

$$
\mathbb{E}[\mathrm{RFF}]=e^{-z^{2} / 2}, \mathbb{V}[\mathrm{RFF}]=\frac{\left(1-e^{-z^{2}}\right)^{2}}{2 D}
$$

Due to $\mathbb{V}\left[R_{m}(f)\right]=\frac{1}{D}\left(\mathbb{V}\left[r_{m}(f)\right]\right)$ with $\boldsymbol{\omega} \sim \mathcal{N}\left(\mathbf{0}, \boldsymbol{I}_{d}\right)$, in the next we focus on $\mathbb{V}\left[r_{m}(f)\right]$. The variance of $r_{m}(f)$ can be formulated as

$$
\begin{aligned}
\mathbb{V}\left[r_{m}(f)\right] & =\mathbb{E}\left[f(\boldsymbol{\omega})-M^{(m, d)}(f)+Q^{(m, d)}(f)\right]^{2}-\left\{\mathbb{E}\left[f(\boldsymbol{\omega})-M^{(m, d)}(f)+Q^{(m, d)}(f)\right]\right\}^{2} \\
& =\mathbb{E}\left[f(\boldsymbol{\omega})-M^{(m, d)}(f)+Q^{(m, d)}(f)\right]^{2}-\{\mathbb{E}[f(\boldsymbol{\omega})]\}^{2} \\
& =\mathbb{E}\left[f(\boldsymbol{\omega})-M^{(m, d)}(f)+Q^{(m, d)}(f)\right]^{2}-e^{-z^{2}},
\end{aligned}
$$

where the used Gaussian kernel admits $f(\boldsymbol{\omega})=\cos \left(\boldsymbol{\omega}^{\top} \boldsymbol{z}\right)$.

Further, the above equation can be rewritten as

$$
\begin{aligned}
\mathbb{V}\left[r_{m}(f)\right]= & \mathbb{E}\left[f^{2}(\boldsymbol{\omega})\right]+\mathbb{E}\left[\left(M^{(m, d)}(f)\right)^{2}\right]+\left[Q^{(m, d)}(f)\right]^{2}+2 \mathbb{E}\left[f(\boldsymbol{\omega}) Q^{(m, d)}(f)\right]-2 \mathbb{E}\left[f(\boldsymbol{\omega}) M^{(m, d)}(f)\right] \\
& -2 \mathbb{E}\left[M^{(m, d)}(f) Q^{(m, d)}(f)\right]-e^{-z^{2}} \\
= & D \mathbb{V}[\mathrm{RFF}]-\left[Q^{(m, d)}(f)\right]^{2}+2 e^{-z^{2} / 2} Q^{(m, d)}(f)+\mathbb{E}\left[\left(M^{(m, d)}(f)\right)^{2}\right]-2 \mathbb{E}\left[f(\boldsymbol{\omega}) M^{(m, d)}(f)\right] .
\end{aligned}
$$

Hence, we need to bound $\mathbb{E}\left[\left(M^{(m, d)}(f)\right)^{2}\right]$ and $\mathbb{E}\left[f(\boldsymbol{\omega}) M^{(m, d)}(f)\right]$ in Eq. (B.6).

First, for our third-degree rule, we estimate $\mathbb{E}\left[\left(M^{(m, d)}(f)\right)^{2}\right]$ by expanding $\left[M^{(m, d)}(f)\right]^{2}$ in Eq. (4.1) with $m=1$

$$
\begin{aligned}
{\left[M^{(1, d)}(f)\right]^{2} } & =\left(1-\frac{\sum_{i=1}^{d} \omega_{i}^{2}}{\lambda_{1}^{2}}\right)^{2}+\frac{\left(\sum_{i=1}^{d} \omega_{i}^{2}\right)^{2}}{4 \lambda_{1}^{4} d^{2}}\left\{\sum_{i=1}^{d}[f]\right\}^{2}+\left(1-\frac{\sum_{i=1}^{d} \omega_{i}^{2}}{\lambda_{1}^{2}}\right) \frac{\sum_{i=1}^{d} \omega_{i}^{2}}{\lambda_{1}^{2} d} \sum_{i=1}^{d}[f] \\
& =1-\frac{2 \sum_{i=1}^{d} \omega_{i}^{2}}{\lambda_{1}^{2}}+\frac{\left(\sum_{i=1}^{d} \omega_{i}^{2}\right)^{2}}{\lambda_{1}^{4}}+\frac{\left(\sum_{i=1}^{d} \omega_{i}^{2}\right)^{2}}{4 \lambda_{1}^{4} d^{2}}\left\{\sum_{i=1}^{d}[f]\right\}^{2}+\frac{\sum_{i=1}^{d} \omega_{i}^{2}}{\lambda_{1}^{2} d} \sum_{i=1}^{d}[f]-\frac{\left(\sum_{i=1}^{d} \omega_{i}^{2}\right)^{2}}{\lambda_{1}^{4} d} \sum_{i=1}^{d}[f],
\end{aligned}
$$

where we use $f(\mathbf{0})=1$. Accordingly, the expectation of $\left[M^{(1, d)}(f)\right]^{2}$ is

$$
\mathbb{E}\left[M^{(1, d)}(f)\right]^{2}=1-\frac{2 d}{\lambda_{1}^{2}}+\frac{d^{2}+2 d}{\lambda_{1}^{4}}+\frac{d+2}{4 \lambda_{1}^{4} d}\left\{\sum_{i=1}^{d}[f]\right\}^{2}+\frac{1}{\lambda_{1}^{2}} \sum_{i=1}^{d}[f]-\frac{d+2}{\lambda_{1}^{4}} \sum_{i=1}^{d}[f]
$$


where we use $\sum_{i=1}^{d} \omega_{i}^{2} \sim \chi(d)$. Also, we have $\mathbb{E}\left(\sum_{i=1}^{d} \omega_{i}^{2}\right)=d, \mathbb{V}\left(\sum_{i=1}^{d} \omega_{i}^{2}\right)=2 d$ and $\mathbb{E}\left(\left[\sum_{i=1}^{d} \omega_{i}^{2}\right]^{2}\right)=d^{2}+2 d$.

Second, we estimate $-2 \mathbb{E}\left[f(\boldsymbol{\omega}) M^{(1, d)}(f)\right]$. The notation $f(\boldsymbol{\omega}) M^{(1, d)}(f)$ is formulated as

$$
f(\boldsymbol{\omega}) M^{(1, d)}(f)=\left(1-\frac{\sum_{i=1}^{d} \omega_{i}^{2}}{\lambda_{1}^{2}}\right) f(\boldsymbol{\omega})+\frac{\sum_{i=1}^{d} \omega_{i}^{2}}{2 \lambda_{1}^{2} d} f(\boldsymbol{\omega}) \sum_{i=1}^{d}[f] .
$$

Accordingly, we have

$$
-2 \mathbb{E}\left[f(\boldsymbol{\omega}) M^{(1, d)}(f)\right]=-2 e^{-z^{2} / 2}+\frac{2}{\lambda_{1}^{2}} \mathbb{E}\left[f(\boldsymbol{\omega}) \sum_{i=1}^{d} \omega_{i}^{2}\right]-\frac{\sum_{i=1}^{d}[f]}{\lambda_{1}^{2} d} \mathbb{E}\left[f(\boldsymbol{\omega}) \sum_{i=1}^{d} \omega_{i}^{2}\right],
$$

where we use $f(\mathbf{0})=1$ and $\mathbb{E}[f(\boldsymbol{w})]=e^{-z^{2} / 2}$. Hence, combining Eqs. (B.6), (B.7), (B.8) and Lemma B.1, the variance of $r_{m}(f)$ with $m=1$ is

$$
\begin{aligned}
\mathbb{V}\left[r_{1}(f)\right]-D \mathbb{V}[\mathrm{RFF}] & =1-\frac{d}{\lambda_{1}^{2}}+\frac{d^{2}+2 d}{\lambda_{1}^{4}}+\frac{d+2}{4 \lambda_{1}^{4} d}\left\{\sum_{i=1}^{d}[f]\right\}^{2}+\frac{1}{\lambda_{1}^{2}} \sum_{i=1}^{d}[f]-\frac{d+2}{\lambda_{1}^{4}} \sum_{i=1}^{d}[f] \\
& -\left[Q^{(1, d)}(f)\right]^{2}+2 e^{-z^{2} / 2} Q^{(1, d)}(f)-2 e^{-z^{2} / 2}+\frac{1}{\lambda_{1}^{2}}\left(2-\frac{\sum_{i=1}^{d}[f]}{d}\right) e^{-\frac{z^{2}}{2}}\left(d-z^{2}\right) .
\end{aligned}
$$

Due to $\sum_{i=1}^{d}[f]=2 \lambda_{1}^{2} Q-2 \lambda_{1}^{2}+2 d$ in the third-degree fully symmetric interpolatory rule, we have

$$
\mathbb{E}\left[M^{(1, d)}(f)\right]^{2}=1+\frac{d+2}{d}(Q-1)^{2}+2(Q-1),
$$

and

$$
-2 \mathbb{E}\left[f(\boldsymbol{\omega}) M^{(1, d)}(f)\right]=-\frac{2(Q-1)}{d} e^{-\frac{z^{2}}{2}}\left(d-z^{2}\right) .
$$

Hence, combing the above two equations, Eq. (B.9) can be further improved as

$$
\begin{aligned}
\mathbb{V}\left[R_{1}(f)\right]-\mathbb{V}[\mathrm{RFF}] & =\frac{2}{D d}\left((1-Q)^{2}-(1-Q) z^{2} e^{-\frac{z^{2}}{2}}\right) \\
& =\frac{2}{D d}\left(\left[(1-Q)-\frac{1}{2} z^{2} e^{-\frac{z^{2}}{2}}\right]^{2}-\frac{1}{4} z^{4} e^{-z^{2}}\right) .
\end{aligned}
$$

Since $Q:=Q^{(1, d)}(f)$ is the approximation of $I_{d}(f)=k(\boldsymbol{x}, \boldsymbol{y}) \in[0,1]$ for the Gaussian kernel, we can also consider $Q^{(1, d)}(f) \in[0,1]$. Note that, even if the estimation $Q^{(1, d)}(f)$ is out of $[0,1]$, we can still set it to $[0,1]$ by a threshold operator and thus $1-Q \geq 0$. Finally, Eq. (B.10) can be formulated as

$$
\mathbb{V}\left[R_{1}(f)\right]-\mathbb{V}[\mathrm{RFF}]<0 \text { when } 1-Q<z^{2} e^{-\frac{z^{2}}{2}},
$$

which concludes the proof.

\section{B.2.3 Condition verification of Theorem 2}

For the Gaussian kernel, denote $Q:=k(\boldsymbol{x}, \boldsymbol{y})+\epsilon=e^{-\frac{z^{2}}{2}}+\epsilon$ with a residual error $\epsilon$, then the used condition $1-Q<z^{2} e^{-\frac{z^{2}}{2}}$ in Theorem 2 is equivalent to

$$
\epsilon>1-\left(z^{2}+1\right) e^{-\frac{z^{2}}{2}}=: J(z)
$$




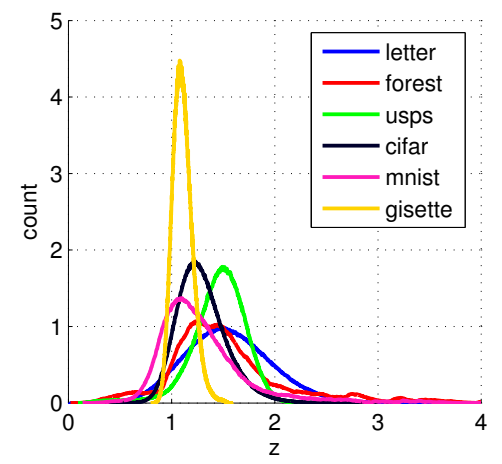

(a) Empirical distribution of $z$

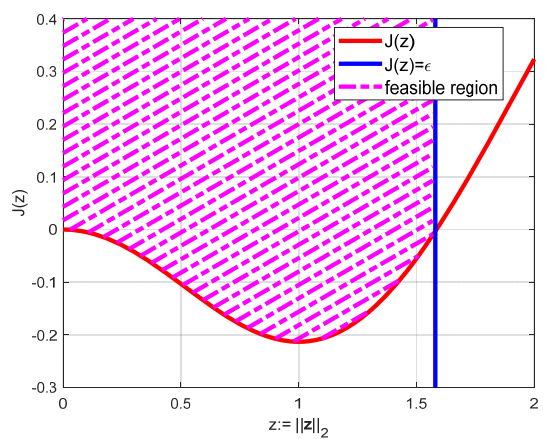

(b) $J(z)$

Figure 9: Empirical distribution of $z$ in (a) and $J(z)$ in (b).

In the next, we investigate the empirical distribution of $z$ and plot $J(z)$ to find the feasible region for $\epsilon$.

Figure 9(a) is given by [YSC $\left.{ }^{+} 16\right]$ (see Figure 2(c) in their paper), which shows distribution of $z$ for several datasets. The count is normalized such that the area under curve for each dataset is 1 . Observe that most nodes in all the datasets have $z<2$. Based on this, we plot $J(z)$ versus $z$ on a range of $[0,2]$ in Figure 9 (b) to show how many area admits $\epsilon>J(z)$. Since quadrature based methods have high approximation quality to approximate the $d$-dimensional integral in Eq. (1.1), the fully symmetric interpolatory rule admits an extremely small residual error around 0 . If we set $\epsilon$ to zero, we can get the feasible region (see in pink) satisfying $\epsilon>J(z)$ in Figure $9(\mathrm{~b})$. Note that $J(z)=0$ indicates $z \approx 1.57$ (i.e., the blue curve), then Eq. (B.11) holds when $z$ approximately satisfies $z \leq 1.57$. Hence, from Figure 9, we can conclude that Eq. (B.11) holds for most cases based on the empirical distribution of $z$. More importantly, if we normalize $z:=\|\boldsymbol{x}-\boldsymbol{y}\| / \sigma$ to $z:=\|\boldsymbol{x}-\boldsymbol{y}\| / \sqrt{d \sigma^{2}}$ by a scaling factor $\sqrt{d}$, the condition $z \leq 1.57$ can be easily satisfied. This operation is fair since it is quite common in high-dimensional statistics and random matrix theory [EK10, DW18, LR20], and our experiment employs this setting.

Note that even if the scaled $z$ can not guarantee $z \leq 1.57$, we cannot obtain $\mathbb{V}\left[R_{1}(f)\right]-\mathbb{V}[\mathrm{RFF}]<0$ but we still have

$$
\mathbb{V}\left[R_{1}(f)\right]-\mathbb{V}[\mathrm{RFF}] \leq \frac{2}{D d} .
$$

As a result, $\mathbb{V}\left[R_{1}(f)\right]$ is asymptotically smaller than that of RFF when $D d$ is large, which is often satisfied in many real-world datasets.

\section{Proof of Theorem 3}

To prove Theorem 3, we need the following lemma.

Lemma C.1. (Theorem 4.1 in [JXC15]) Denote $\boldsymbol{x}^{M}$ and $\boldsymbol{s}^{M}$ as polynomials with total degree $M$, then the following integral satisfies

$$
I^{\prime}\left(\boldsymbol{\omega}^{d}\right)=\int_{\mathbb{R}^{n}} \omega_{1}^{\alpha_{1}} \omega_{2}^{\alpha_{2}} \cdots \omega_{d}^{\alpha_{d}} \exp \left(-\boldsymbol{\omega}^{\top} \boldsymbol{\omega}\right) \mathrm{d} \boldsymbol{x}=\int_{0}^{\infty} r^{d-1+M} \exp \left(-r^{2}\right) \mathrm{d} r \int_{U_{d}} \boldsymbol{s}^{M} \mathrm{~d} \sigma(\boldsymbol{s}),
$$

can be exactly calculated by the quadrature rules $I^{\prime}(\boldsymbol{\omega})=\sum_{j=1}^{N_{g}} \bar{a}_{j} f(\bar{\gamma})$ with $f^{\prime}(\boldsymbol{\omega})=\boldsymbol{\omega}^{M}$. Then the spherical integral can be expressed as $\int_{U_{d}} \boldsymbol{s}^{M} \mathrm{~d} \sigma(\boldsymbol{s})=\sum_{j=1}^{N_{p}} a_{s, j}\left(\mathrm{~s}_{j}\right)^{M}$ with the nodes $\boldsymbol{s}_{j}=\frac{\overline{\boldsymbol{\gamma}}_{j}}{\left\|\overline{\boldsymbol{\gamma}}_{j}\right\|_{2}}$ and and the weights $a_{s, j}$ of the spherical rule are

$$
a_{s, j}=\frac{\bar{a}_{j}\left(\boldsymbol{s}_{j}\right)^{M}}{\int_{0}^{\infty} r^{d-1+M} \exp \left(-r^{2}\right) \mathrm{d} r}=\frac{\bar{a}_{j}\left(\boldsymbol{s}_{j}\right)^{M}}{\Gamma(d / 2+M / 2) / 2},
$$

where $N_{p}$ is the number of projected quadrature non-zero nodes. Note that $N_{p} \leq N_{g}$. 
Formally, we are ready to prove Theorem 3.

Proof The integral in Eq. (1.1) can be reformulated as

$$
I_{d}\left(f_{\boldsymbol{x} \boldsymbol{y}}\right)=\int_{\mathbb{R}^{d}} f_{\boldsymbol{x} \boldsymbol{y}}(\boldsymbol{\omega}) \mathcal{N}\left(\boldsymbol{\omega} ; \mathbf{0}, \boldsymbol{I}_{d}\right) \mathrm{d} \boldsymbol{\omega}=\pi^{-\frac{d}{2}} \int_{\mathbb{R}^{d}} e^{-\boldsymbol{\omega}^{\top} \boldsymbol{\omega}} f(\sqrt{2} \boldsymbol{\omega}) \mathrm{d} \boldsymbol{\omega} .
$$

Hence, the integral $I^{\prime}(\boldsymbol{\omega})=\int_{\mathbb{R}^{d}} f(\boldsymbol{\omega}) \exp \left(-\boldsymbol{\omega}^{\top} \boldsymbol{\omega}\right) \mathrm{d} \boldsymbol{\omega}$ can be approximated by our third-degree fully symmetric interpolatory rules in Eq. (3.2), that is

$$
\begin{aligned}
& I^{\prime}(\boldsymbol{\omega})=\pi^{\frac{d}{2}} \int_{\mathbb{R}^{d}} f\left(\frac{\boldsymbol{\omega}}{\sqrt{2}}\right) \mathcal{N}\left(\boldsymbol{\omega} ; \mathbf{0}, \boldsymbol{I}_{d}\right) \mathrm{d} \boldsymbol{\omega} \approx \sum_{i=1}^{2 d+1} \bar{a}_{i} f\left(\overline{\boldsymbol{\gamma}}_{i}\right) \\
& =\left(1-\frac{d}{\lambda_{1}^{2}}\right) \pi^{\frac{d}{2}} f(\mathbf{0})+\frac{\pi^{\frac{d}{2}}}{2 \lambda_{1}^{2}} \sum_{i=1}^{d}\left(f\left(\frac{\lambda_{1}}{\sqrt{2}} \boldsymbol{e}_{i}\right)+f\left(-\frac{\lambda_{1}}{\sqrt{2}} \boldsymbol{e}_{i}\right)\right),
\end{aligned}
$$

with

$$
\left\{\begin{array}{l}
\bar{\gamma}_{i}=\mathbf{0} ; \bar{a}_{i}=\left(1-\frac{d}{\lambda_{1}^{2}}\right) \pi^{\frac{d}{2}} ; i=0 \\
\bar{\gamma}_{i}=\frac{\lambda_{1}}{\sqrt{2}} \boldsymbol{e}_{i} ; \bar{a}_{i}=\frac{\pi^{\frac{d}{2}}}{2 \lambda_{1}^{2}} ; 1 \leq i \leq d \\
\bar{\gamma}_{i}=-\frac{\lambda_{1}}{\sqrt{2}} \boldsymbol{e}_{i-d} ; \bar{a}_{i}=\frac{\pi^{\frac{d}{2}}}{2 \lambda_{1}^{2}} ; d+1 \leq i \leq 2 d .
\end{array}\right.
$$

By projecting $\bar{\gamma}_{i}$ on the surface of the unit $U_{d}$ sphere with an uniform random orthogonal matrix $\boldsymbol{Q}$, we have

$$
\boldsymbol{s}_{i}=\frac{\boldsymbol{Q} \overline{\boldsymbol{\gamma}}_{i}}{\left\|\boldsymbol{Q} \bar{\gamma}_{i}\right\|_{2}^{2}}=\left\{\begin{array}{l}
\boldsymbol{Q} \boldsymbol{e}_{i} ; 1 \leq i \leq d \\
-\boldsymbol{Q} \boldsymbol{e}_{i-d} ; d+1 \leq i \leq 2 d
\end{array}\right.
$$

with $\left\|\boldsymbol{Q} \bar{\gamma}_{i}\right\|_{2}=\left\|\bar{\gamma}_{i}\right\|_{2}$. Note that the point at the origin has been omitted. By Lemma C.1, for the thirddegree, the polynomial degree $M$ is set to 2 . Accordingly, the weight $a_{s, j}$ of the spherical rule can be obtained by

$$
a_{s, j}=\frac{\bar{a}_{j}\left(\boldsymbol{s}_{j}\right)^{M}}{\Gamma(d / 2+M / 2) / 2}=\frac{\pi^{\frac{d}{2}}}{2 \lambda_{1}^{2}} \frac{\lambda_{1}^{2}}{2} /\left(\frac{\frac{d}{2} \Gamma\left(\frac{d}{2}\right)}{2}\right)=\frac{\left|U_{d}\right|}{2 d} .
$$

Hence, using Eq. (C.1) and Eq. (C.2) yields the spherical rule

$$
I_{\boldsymbol{Q}, U_{d}}(s)=\frac{\left|U_{d}\right|}{2 d} \sum_{j=1}^{d}\left[s\left(\boldsymbol{Q} \boldsymbol{e}_{j}\right)+s\left(-\boldsymbol{Q} \boldsymbol{e}_{j}\right)\right],
$$

which is identical to the third-degree stochastic spherical integration rule in Eq. (5.2). 